\title{
Improving Radar Rainfall Estimation by Accounting for Microphysical Processes Using a Micro Rain Radar in West Africa
}

\section{Ghislain Kouadio, Eric-Pascal Zahiri*, Modeste Kacou, Augustin Kadjo Koffi, Abé Delfin Ochou, Paul Assamoi}

Laboratoire de Physique de l'Atmosphère et de Mécanique des Fluides (LAPA-MF), UFR SSMT, Université Félix Houphouët-Boigny, Abidjan, Côte d'Ivoire

Email: ^zahiripascal@gmail.com, *eric.zahiri@univ-fhb.edu.ci

How to cite this paper: Kouadio, G., Zahiri, E.-P., Kacou, M., Koffi, A.K., Ochou, A.D. and Assamoi, P. (2021) Improving Radar Rainfall Estimation by Accounting for Microphysical Processes Using a Micro Rain Radar in West Africa. Atmospheric and Climate Sciences, 11, 658-688.

https://doi.org/10.4236/acs.2021.114039

Received: June 1, 2021

Accepted: August 29, 2021

Published: September 1, 2021

Copyright $\odot 2021$ by author(s) and Scientific Research Publishing Inc. This work is licensed under the Creative Commons Attribution International License (CC BY 4.0).

http://creativecommons.org/licenses/by/4.0/

(c) (i) Open Access

\begin{abstract}
This study evaluates the improvement of the radar Quantitative Precipitation Estimation (QPE) by involving microphysical processes in the determination of $Z-R$ algorithms. Within the framework of the AMMA campaign, measurements of an X-band radar (Xport), a vertical pointing Micro Rain Radar (MRR) to investigate microphysical processes and a dense network of rain gauges deployed in Northern Benin (West Africa) in 2006 and 2007 were used as support to establish such estimators and evaluate their performance compared to other estimators in the literature. By carefully considering and correcting MRR attenuation and calibration issues, the $Z$ - $R$ estimator developed with the contribution of microphysical processes and non-linear least-squares adjustment proves to be more efficient for quantitative rainfall estimation and produces the best statistic scores than other optimal $Z-R$ algorithms in the literature. We also find that it gives results comparable to some polarimetric algorithms including microphysical information through DSD integrated parameter retrievals.
\end{abstract}

\section{Keywords}

Drop Distribution, Micro Rain Radar, Calibration, Microphysical Processes, $Z-R$ Relationships, Rainfall Estimation

\section{Introduction}

An important step in the quantitative estimation of rain by radar is the determination of a suitable algorithm to efficiently transform radar information into rain characteristics. Due to its easy implementation, the relationship $Z=A * R^{b}$ 
between radar reflectivity $(Z)$ and rain rate $(R)$ is the most widely used in the literature when solving attenuation and calibration problems affecting the measurement of reflectivity. However, the main limitation of this algorithm is attributed to the raindrop size distribution (DSD) variability within the rainfall and thus to the diversity of microphysical processes involved in the formation of these raindrops [1].

In the absence of direct consideration of the mechanisms underlying the variability of raindrops and aiming to take into account the fluctuating nature of the DSD in rain estimators design, several authors have favored either an event-byevent study [2] [3] or a classification according to the nature of the precipitation [3]-[8], either by season [9] [10] or by taking into account the maritime or continental origin of the convective precipitation system [11]. These approaches are implicit ways of taking into account the dominant microphysical processes within each precipitation system or specific parts of these convective rainy systems. However, as discussed by [12] and [13], for rain rates considered separately as stratiform or convective, there is great variability in the relationships determined by considering only one type of rainfall. The reason for this lies in the non-injective nature of the relationship itself, i.e. a single value of radar reflectivity may correspond to different DSD spectra and rain rate values [13] [14]. These fluctuations can plausibly be attributed to variations in the microphysical processes underlying the formation of precipitation that change over time the rain rates and convective or non-convective nature of precipitation.

Assuming the DSD data at the ground as a result of physical processes aloft [15] showed that the variability of the coefficients of the $Z-R$ relation is governed by three specific modes of variability, namely the modes associated with the variability of total drop concentration $N_{T}$, drop size through the volume median diameter parameter $D_{0}$ or a combination of both modes through the ratio $\left(D_{0} / N_{T}\right)$. To characterize the variability of the $Z-R$ relationship, [13] suggested that the size (represented by the volume median diameter of the DSD, $D_{0}$ ) and the number of raindrops (represented by $N_{T}$ ) should be considered simultaneously through the ratio that best reflects how they vary with $\mathrm{Z}$ for a given $Z-R$ relationship. Finally, [10] analyzing 12 years of DSD measurements in Northern Taiwan, indicated that the pre-factor A would be sensitive to the presence of drops of large or small drop diameters while the exponent $\mathrm{b}$ determines the microphysical process nature.

Microphysical interpretation of $Z-R$ relationships coefficients seems to be a way to understand and account for their high variability in rainfall estimation. However, involving the temporal fluctuations of the DSD to adjust the dependence of rainfall estimation algorithms on the physical processes prevailing within precipitation systems remains a challenge in the case of the operational use of weather radars [8]. Although the work of [10] [13] [15] shows the interest in taking into account microphysics to improve the quantitative estimation of rainfall by radar, the use of their approach remains difficult to implement in operational use. Moreover, the fact that they are only interested in the raindrop size distri- 
bution at the ground level whereas the DSD fluctuations occur aloft where the radar measurements take place is a handicap. A promising approach to address such type of issues remains the use of a vertically looking radar such as Micro Rain Radar (MRR) to probe raindrop medium aloft and study the vertical variability of the drop size distribution to determine microphysical processes [12] [16] [17] and incorporate their fluctuations into the adjustment of radar rainfall estimation algorithms.

During the intensive AMMA (African Monsoon Multidisciplinary Analysis) campaign in 2006, a Micro Rain Radar (MRR) operated in Northern Benin in addition to two horizontal scanning radars (C-band and X-band) installed to monitor rainfall systems in the region and estimate rainfall over small basins [18] [19], a dense rain gauges network [20] and disdrometers [21]. Such a system offers the opportunity to develop rainfall estimation algorithms by including the microphysical processes that cause rainfall. So far, although the measurements of the other instruments mentioned above have been exploited through different studies [18] [19] [21] [22], the data collected by the MRR during the AMMA campaign have remained unexploited to date. One of the reasons is related to the problems of correcting acute attenuation in K-band [23] for moderate to high rainfall rates encountered in the region and the calibration of the MRR [24] [25] which did not allow the quantitative use of these data. In this study, we propose an approach that exploits the co-location of the different radars and the polarimetry advantages of the X-band radar to develop a method for correcting the absolute calibration of MRR data using a polarimetric variable of the X-band radar, the specific differential phase shift, unaffected by the rain-induced attenuation of the signal. Therefore, the objective of this study includes the correction of the MRR dataset for calibration and attenuation problems for quantitative use in: 1) determining microphysical processes; 2) designing algorithms involving the microphysical processes to improve the estimation of rain by horizontal scanning radar.

The remainder of the paper presents in Section 2, the study area, data and methods for pre-processing the MRR data. Section 3 describes the method for determining dominant microphysical processes and $Z-R$ relationships. Section 4 presents all the results concerning the rainfall estimation from the algorithms including the consideration of microphysical processes and their discussion by comparison to the rainfall estimators with or without consideration of micro-physical processes. Finally, Section 5 is devoted to the conclusion and perspectives of this study.

\section{Data and Experiment Description}

\subsection{General Overview}

The Upper Ouémé Hydro-meteorological observatory AMMA-CATCH (Couplage Atmosphere Tropicale Cycle Hydrologique), depicted in Figure 1, is one of the three mesoscale sites instrumented during AMMA Enhanced Observing Period 


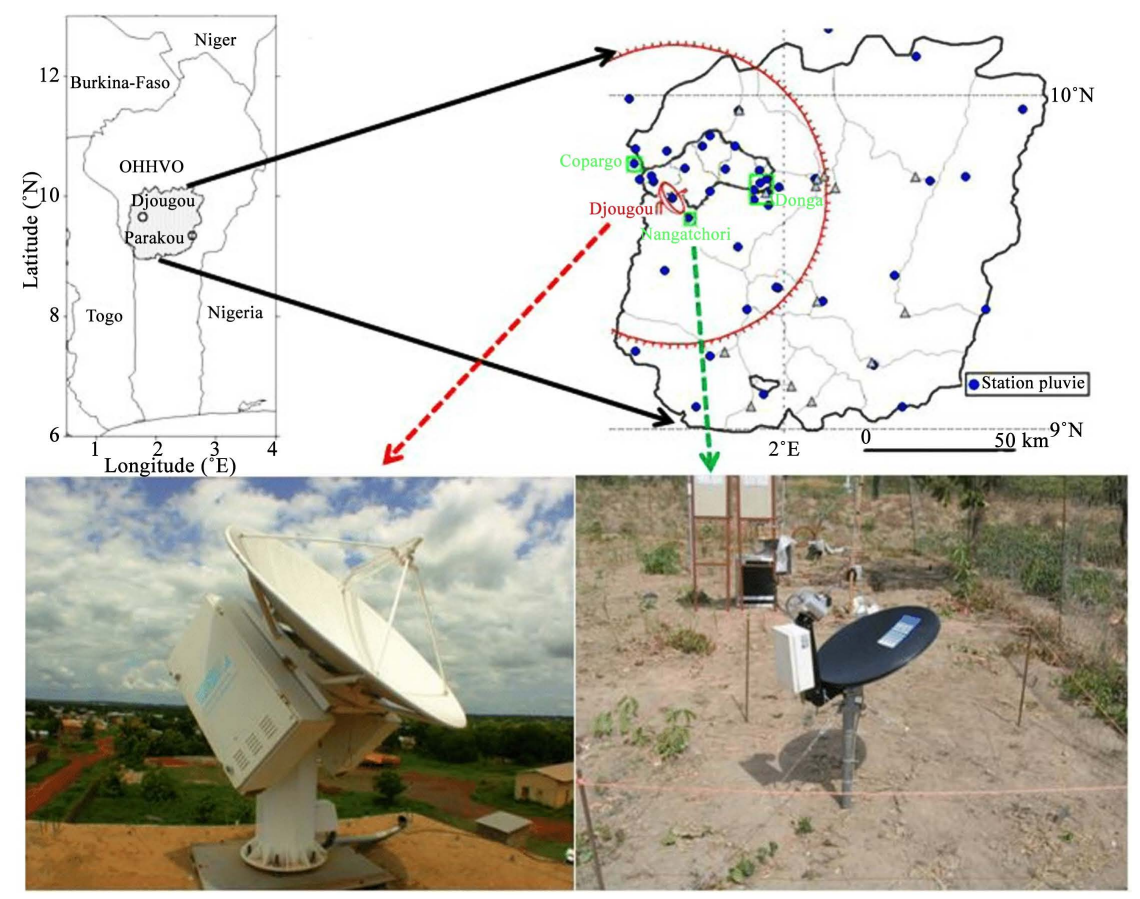

Figure 1. AMMA-CATCH experimental area in Benin showing the measuring instruments installed. Top (on the left) we show a view of Benin (West Africa) and (on the right) the AMMA-CATCH site. Rain gauges are indicated by black dots. Bottom: (on the left), the Xport radar, positioned in Djougou ; (on the right), the Micro Rain Radar (MRR) in Nangatchori (10 km far from radar Xport).

from 2006 to 2007 [26] to analyze the mesoscale convective systems and the rainfall in northern Benin. Radars experiment in that region involved a wide collection of ground-based instruments including rain gauges network of 54 instruments, two horizontal scanning weather radars (C-band radar and X-band radar), a vertically pointing Micro Rain Radar (MRR), and three disdrometers which unfortunately did not regularly operate simultaneously during the intensive campaign because of technical difficulty. The aim of the observational strategy was to provide reliable estimates of rain rates with a very high spatial and temporal resolution. In the present study, mainly details about X-band radar and MRR instruments (shown in Figure 1) and data collected simultaneously by Xport radar (X-band polarimetric radar) and MRR (K-band radar) are concerned because of the unique opportunity given to compare data at the same height levels and thus reduce errors in sampling data even if there are differences in the resolution volumes, which is not the same for both radars. For ground validation of rainfall estimates from radars, rain gauges network dataset is used. The dataset results of 60 rain events, which has been divided into two parts, one for the attenuation correction and the calibration of the MRR, and the other for the validation of the MRR data processing.

\subsection{Description of Experimental Datasets}

Within African rainfall projects, the LTHE (Laboratoire d'Etude des Transferts 
en Hydrologie et Environnement) of Grenoble (France) developed an X-band polarimetric radar (see Figure 1) whose main characteristics were described in [19]. During the AMMA campaign, the X-band polarimetric radar (Xport radar) operated in 2006 and 2007. Its position within the Upper Oueme Hydro-meteorological AMMA-CATCH (Couplage Atmosphere Tropicale Cycle Hydrologique) observatory allows for a full coverage of the Donga basin and others sensors installed such as disdrometers, rain gauges and MRR. With this latter sensor which was installed in Nangatchori (10 km from X-band radar), the Xport radar has a common volume of measurements useful for the MRR calibration and providing valuable information about the quality of that calibration.

Xport radar is X-band dual polarimetric radar. It has a significant advantage over single-polarization systems because it allows multi-parameters measurements using orthogonal polarization. The polarimetric radar observables which were used in this study are the horizontal reflectivity $\left(Z_{H}\right)$ and the specific differential phase shift $\left(K_{D P}\right)$ at low elevations. Reflectivity was corrected from rain attenuation based on the processing carried out in [19].

The MRR (Micro Rain Radar) is vertical pointing K-band radar that operates using the FM-CW (Frequency Modulated Continuous Wave) technique, i.e. it emits a continuous, frequency modulated wave [27]. It is used to measure raindrop size distribution profiles and thus to deduce those of all parameters useful for precipitation characterization. Its main characteristics are summarized in Table 1. It was installed at Nangatchori $\left(9.64^{\circ} \mathrm{N} ; 1.74^{\circ} \mathrm{E}\right)$ and operated only in 2006 during the Special Observation Period (SOP) in the middle of the Enhanced

Table 1. Main technical characteristics of the Micro Rain Radar (MRR).

\begin{tabular}{|c|c|}
\hline \multicolumn{2}{|c|}{ Transmitter } \\
\hline Frequency & $24 \mathrm{GHz}$ \\
\hline Modulation band & $1.5 \mathrm{MHz}$ \\
\hline Transmitter power & $50 \mathrm{~mW}$ \\
\hline \multicolumn{2}{|c|}{ Antenna } \\
\hline Diameter & $60 \mathrm{~cm}$ \\
\hline Beamwidth to $6 \mathrm{~dB}$ & $2^{\circ}$ \\
\hline Elevation & Vertically pointing \\
\hline \multicolumn{2}{|c|}{ Acquisition } \\
\hline Sampling frequency & $125 \mathrm{kHz}$ \\
\hline Temporal resolution & $10 \mathrm{~s}$ \\
\hline Range resolution & $100 \mathrm{~m} / 160 \mathrm{~m}$ \\
\hline Number of gates & 30 \\
\hline Spectral velocity resolution & $0.191 \mathrm{~m} / \mathrm{s}$ \\
\hline Number of bins (Maximal velocity) & $64(12.3 \mathrm{~m} / \mathrm{s})$ \\
\hline Scanning Protocol & Volume scans \\
\hline
\end{tabular}


Observation Period (EOP) of AMMA. The data collected by the MRR was obtained from a spectrum of droplet fall velocity from 0 to $12 \mathrm{~m} \cdot \mathrm{s}^{-1}$ over 64 classes for 30 radar gates with a radial resolution of $160 \mathrm{~m}$ every $10 \mathrm{~s}$. The processing chain of MRR measurements up to the deduction of the raindrop size distributions is performed by the software provided by the manufacturer. The spectral reflectivity is dynamically analyzed to estimate and remove spurious noise in measurements. Then, the relation of [28] is used to convert the falling speed of raindrops, corrected for the variation of air density with altitude, into diameter $\mathrm{D}$ $(\mathrm{mm})$. The analyzed velocity range is limited to velocities from $0.76 \mathrm{~m} \cdot \mathrm{s}^{-1}$ to 9.36 $\mathrm{m} \cdot \mathrm{s}^{-1}$ corresponding to a diameter range from $0.246 \mathrm{~mm}$ to $5.8 \mathrm{~mm}$. Thus, assuming that the raindrops are flattened spheroids of equivalent diameter, the effective backscattering cross-section of a single raindrop calculated according to Mie theory was used to obtain the corresponding raindrop size distribution profile (DSD). Finally, raindrop size distribution provides the radar reflectivity factor $Z\left(\mathrm{~mm}^{6} \cdot \mathrm{m}^{-3}\right)$, the rain rate $R\left(\mathrm{~mm} \cdot \mathrm{h}^{-1}\right)$ and the specific attenuation coefficient $k\left(\mathrm{~dB} \cdot \mathrm{km}^{-1}\right)$.

The characteristic precipitation parameters deduced from MRR, used in this study, were calculated from spectra recorded in $10 \mathrm{~s}$ time resolution for all the events observed at Nangatchori (10 km far from Xport radar). Its include radar reflectivity, precipitation rate, median volume diameter [29], total raindrop concentration (DSD 0 -order moment) and the ratio $D_{0} / N_{T}$. These quantities were then averaged in integration time of $1 \mathrm{~min}$ and $5 \mathrm{~min}$ respectively to be consistent with the time resolution of Xport radar measurements.

During the AMMA campaign, 54 tipping bucket rain gauges were deployed in 2006 and 2007 at the AMMA-CATCH Benin site to carry out hydrological studies. In this work, only the data recorded by the forty rain gauges located less than $50 \mathrm{~km}$ away from the Xport radar were considered for the different validations and comparisons. The data recorded by the five rain gauges of the Nangatchori super site, located $10 \mathrm{~km}$ from the Xport radar, were particularly concerned for the validation of the MRR measurements. All the data from rain gauges were sampled in 5-min resolution as explained in [30].

\section{MRR Data Processing Chain}

\subsection{Rain Attenuation Correction}

Very high-frequency radars such as the MRR, are confronted with large attenuations of the radar signal for precipitation rates between $0.5 \mathrm{~mm} / \mathrm{h}$ and $100 \mathrm{~mm} / \mathrm{h}$ [31]. As far as gas attenuation is concerned, it can be neglected, provided that quantitative interest is given only to corresponding measurements at altitudes below $1500 \mathrm{~m}$. But, attenuation due to rain must be considered regardless of the altitude of measurements. Therefore, rain attenuation is an important issue that needs careful evaluation for quantitative use of data from this type of radar operating at very high frequencies. The steps for rain attenuation correction of MRR data are based on the method detailed in [23] which is an improvement of the 
algorithm of [32]. For this purpose, in order to correct the MRR data for rain attenuation, the attenuation coefficient $k$ was derived from the knowledge of the raindrop size distribution restored from the Doppler spectrum.

\subsection{MRR Calibration Correction Method}

The measurements of the Xport radar installed in Djougou during the intensive phase of the AMMA campaign have been validated through different studies [18] [19]. Indeed, these works showed that the deduced rainfall rates and DSD parameters retrieved by the Xport radar have a good agreement with the measurements of rain gauges and disdrometers. Thus, the Xport radar measurements could be considered as a reference that can be used to calibrate the MRR first and then to verify its performances. To this end, simultaneous measurements of Xport and the MRR (located in Nangatchori at $10 \mathrm{~km}$ from Xport position) were used to make quantitative comparisons. The Xport radar performs a horizontal scan protocol at different elevations while the MRR scanning protocol is vertical. Thus, due to their respective positioning and scanning protocol, the Xport and MRR radars have a common volume of beam overlap. Furthermore, due to $0.7^{\circ}$ and $3^{\circ}$ of Xport elevation angle and the MRR located at $10 \mathrm{~km}$ from the Xport, the theoretical gate numbers corresponding to this common volume are gate 67 for the Xport and gates 2 and 3 for the MRR. This configuration offers the possibility to compare the identical variables derived from the two radars at the same altitude. This overcomes the difficulty of comparing the same variable from both radars measurements. Therefore, to calibrate the MRR, the simultaneous measurements from the MRR and the Xport at the same altitude were compared. Specifically, the specific differential phase shift $K_{D P}$ that is independent of the effect of attenuation and partial beam occlusion was used for the absolute calibration of the MRR.

In this study, the reflectivity bias due to a calibration error is determined with a method based on consistency between different polarimetric variables. This method is in essence close to the extended Kalman Filter [33]. The principle is based on the fact that the different polarimetric variables can be estimated from each other. For example, $K_{D P}$ is estimated from a linear (logarithmic scale) relationship with the unattenuated horizontal reflectivity $Z_{H}$ [19] [33] [34]. Thus, using the disdrometer data gathered during the AMMA campaigns and radar variable simulations with the T-Matrix, [19] suggested the following relationship:

$$
Z_{e H}=45.0+12.02 \times \log _{10}\left(K_{D P}\right)
$$

where $Z_{e H}$ is in dBZ. This relationship is obtained by assuming [35] raindrop shape model and considering a temperature of $25^{\circ} \mathrm{C}$.

From Equation (1), theoretical $K_{D P}$ values are calculated from different offsets added on the MRR radar reflectivity factor corrected for attenuation. For implementation, offset values ranging from $-20 \mathrm{dBZ}$ to $20 \mathrm{dBZ}$ in steps of $0.01 \mathrm{dBZ}$ are used. By comparison of the theoretical $K_{D P}$ with the intrinsic values derived 
from Xport in the common MRR-Xport sample volume, the offset determination is associated with the lowest value of the absolute specific differential phase shift bias.

\subsection{Statistic Metrics for Evaluation}

The quality of attenuation and calibration correction and estimators for quantitative precipitation estimation is performed using performance criteria such as normalized bias (NB), normalized root mean square error (NRMSE), and Nash Sutcliffe Efficiency. As a reminder, the NRMSE less well known to all others is given by Equation (2):

$$
\text { NRMSE }=\sqrt{\sum_{i=1}^{N}\left(e_{i}-m_{i}\right)^{2}} / \sqrt{\sum_{i=1}^{N} m_{i}^{2}}
$$

with, $m$ standing for the observations and $e$ indicating radar rainfall estimates.

Performance criteria calculations and comparisons were done exclusively with MRR data at the $480 \mathrm{~m}$ altitude (third gate and first valid gate in the MRR measurements). Indeed, the data from the first two gates are spurious as they are not quantitatively exploitable [36] and are not considered in future analyses.

\subsection{Dominant Microphysical Processes and Z-R Relationship Determination}

The microphysical processes taking place at altitude are responsible for the $N(D)$ distribution of the raindrops observed at the ground. Reference [37] showed that microphysical processes such as coalescence, breakup, combination of coalescence and breakup, collection, evaporation, updraft, downdraft, and sorting of raindrops act together or alone to form the actual distribution of drops. Reference [15] suggested that the shape of the raindrop size distribution (DSD) is governed by variations in raindrop concentration and size and by the combined variation of these two parameters. Thus, based on the works of [15] [16] [37], the various dominant microphysical processes that modulate the shape of the DSD were determined using DSD profiles measured by the MRR and averaged over a 5-minutes time step.

The raindrop distributions ratio $N(D)_{\text {test }} / N(D)_{\text {ref }}$ at different altitudes and their evolutions are analyzed to determine the most plausible processes responsible for DSD. $N(D)_{\text {ref }}$ refers to the distribution of raindrops at the reference altitude of $1120 \mathrm{~m}$ (sixth gate of the MRR) considered as close to the base of the cloud generally located at $1000 \mathrm{~m}$ in this region. $N(D)_{\text {test }}$ is the distribution of raindrops at a given altitude outside the reference altitude. For the discrimination of microphysical processes, the growth $\left(N(D)_{\text {test }} / N(D)_{\text {ref }}<1\right)$ or decay $\left(N(D)_{\text {test }} / N(D)_{\text {ref }}>1\right)$ characterizing the DSD at the reference altitude compared to those at higher test altitudes are analyzed for three different classes of drop diameters representing respectively small drops $(D<2 \mathrm{~mm})$, medium drops ( $2 \leq D \leq 4 \mathrm{~mm}$ ) and large drops ( $D>4 \mathrm{~mm}$ ) as proposed in [16]. Thus, based on the classification of [37], the following microphysical processes are 
highlighted:

- The coalescence which decreases the number of small drops and increases the number of larger drops;

- The breakup which causes an increase in the number of small drops and a decrease in those of large drops;

- The coalescence-breakup combination which includes the effects of coalescence and breakup simultaneously;

- Evaporation which causes a greater decrease in the number of small drops than in the number of large drops;

- The updraft which leads to a decrease in the number of small drops at lower altitudes.

- Downdraft which increases the flow of raindrops for any diameter;

- Sorting of drops according to their size, which tends towards a monomodal distribution of drops.

Based on a rainy averaged sequence of 5 minutes per $10 \mathrm{~s}$ sampling step, a microphysical process is associated with each fraction of $N(D)_{\text {test }} / N(D)_{\text {ref }}$ raindrop concentration. Ultimately, the microphysical mechanism of greatest occurrence is related to the entire 5-minutes sequence considered.

In terms of rainfall algorithm, the coefficients $A$ and $b$ of the $Z-R$ relationship are obtained by combining the reflectivity $Z$ deduced from radar and the rain rates $R$ provided by ground rain gauges. Differences in sampling between these two sensors and differences in measurement altitudes may affect the representativeness of these coefficients and the rainfall estimates derived from these algorithms. To circumvent this drawback, other authors use disdrometer measurements to determine coefficients $A$ to $b$, taking advantage of the fact that measurements of raindrop size distributions allows the calculation of rainfall rate and reflectivity from a single instrument. However, the estimates depend on the types of disdrometers used, the mathematical method used to determine these coefficients, and the representativeness of a disdrometer's measurements relative to radar measurements.

$Z-R$ relationships are usually inferred from a linear regression in a semi-log space. Linear regression adjusts a line that minimizes intercept deviations between the measurement points and the fitting line. The line found is therefore not the same depending on whether one or the other variable is placed on the ordinate (dependent variable). Reference [38] showed that the choice of the dependent variable $(Z$ or $R$ ) for establishing the relationships is important since the differences are as large as if one were dealing with different types of precipitation. In addition, [39] indicated that the determination of $Z-R$ coefficients is sensitive to the analytical methods used.

In the present work, the corrected and validated MRR data from which rain rate and reflectivity were derived are used to calculate the pre-factor $A$ and the exponent $b$ of the $Z$ - $R$ relationships using two analytical methods. Thus, with or without taking into account microphysical processes, we have, using the linear 
regression method and the non-linear least squares adjustment method, to determine the various $\mathrm{A}$ and $\mathrm{b}$ coefficients of the $Z-R$ relations at each MRR radar gate, i.e. as a function of altitude.

The $Z$ - $R$ relationship is of the form:

$$
\mathrm{Z}_{\mathrm{MRR}}=A \cdot R_{\mathrm{MRR}}^{b}
$$

where the reflectivity $Z_{\mathrm{MRR}}$ and rain rate $R_{\mathrm{MRR}}$ are deducted from the MRR measurements of DSD. $A$ and $b$ in Equation (3) are obtained by linear regression at the semi-logarithmic scale. For the non-linear least squares fitting method [40], the power law coefficients $A^{\prime}$ and $b^{\prime}$ are calculated by minimizing a cost function that is equivalent to a quadratic deviation of the form:

$$
F\left(A^{\prime}, b^{\prime}\right)=\sum_{i=1}^{M}\left[R_{\mathrm{MRR}}(i)-R(i)\right]^{2}
$$

where, $F$ is the function to be minimized, $i$ indicates the spectrum considered in a 5-minute rainy sequence and $R$ is the theoretical rain rate calculated from the relation:

$$
R=10^{\left(10 \log Z_{\mathrm{MRR}} / 10 b^{\prime}-\log A^{\prime} / b^{\prime}\right)}
$$

$A^{\prime}$ and $b^{\prime}$ are the optimized coefficients of the $Z-R$ relations calculated at each iteration and Equation (5) is obtained from Equation (3). The optimization algorithm thus adopted is a subspace method called "trust region" and is based on Newton's interior-reflective method [41] [42]. The optimized coefficients are calculated iteratively from the coefficients obtained using the linear regression method which are used as the starting point for the iteration procedure.

In total, for each radar gate, four $Z-R$ relations were determined, namely two rainfall estimators taking into account the microphysical processes derived from linear and non-linear regression respectively and two others without taking into account these processes still deduced from the two statistical methods mentioned above. Finally, we analyzed the vertical variability of the $Z-R$ relations by discussing, as a function of the different dominant microphysical processes, the implication of the drop size parameters $D_{0}$, total drop concentration $N_{T}$ and the resulting ratio $D_{0} / N_{T}$.

\section{Results and Discussion}

\subsection{Impact of MRR Data Correction}

Figure 2 shows the absolute bias curve between the intrinsic and theoretical $K_{D P}$ values as a function of the initial bias to compensate for the miscalibration of the reflectivity measured by the MRR. The minimum of the curve shown indicates a bias of $8.08 \mathrm{dBZ}$. Figure 3 shows the contours and profiles of the Xport and MRR radar reflectivity factor before and after the attenuation and calibration corrections, respectively, for the event of 01-02/08/2006. These contours and profiles show a qualitative improvement with a significant increase in the corrected reflectivity values over those uncorrected. Indeed, by comparing these figures, qualitative improvement is obvious showing an increase in the values of corrected reflectivity with respect to uncorrected one. 


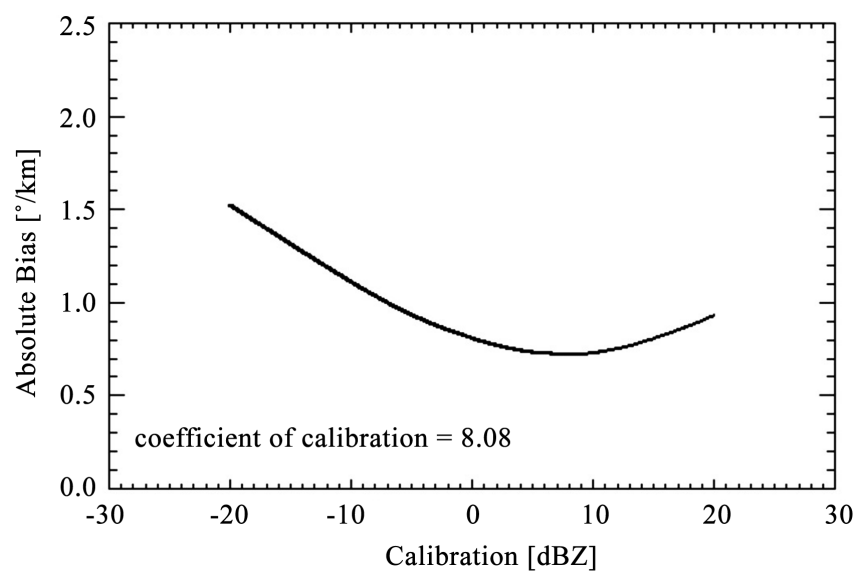

Figure 2. Absolute bias between the directly estimated $K_{d p}$ from Xport radar measurements and et $K_{d p}$ retrieved from MRR using the $K_{d p}-Z_{h}$ relationship for different reflectivity correction values.
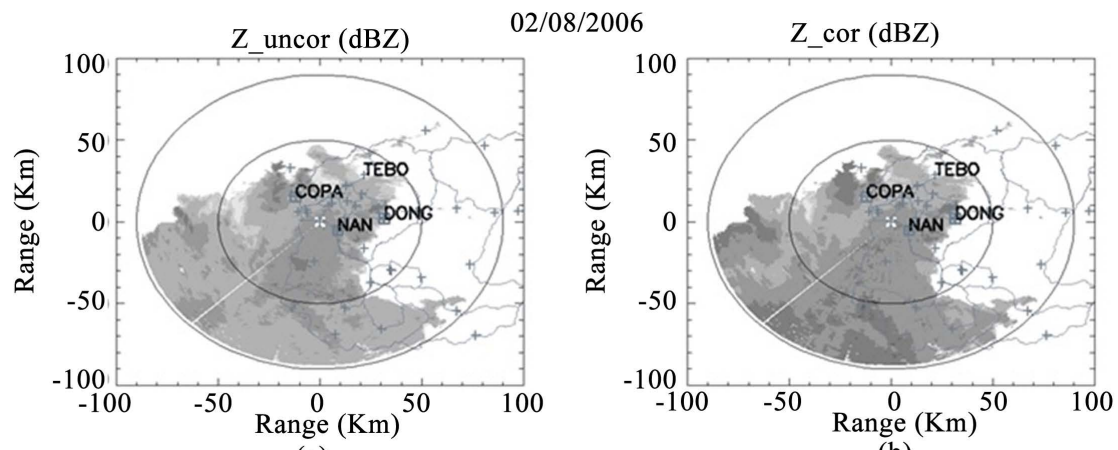

(a)
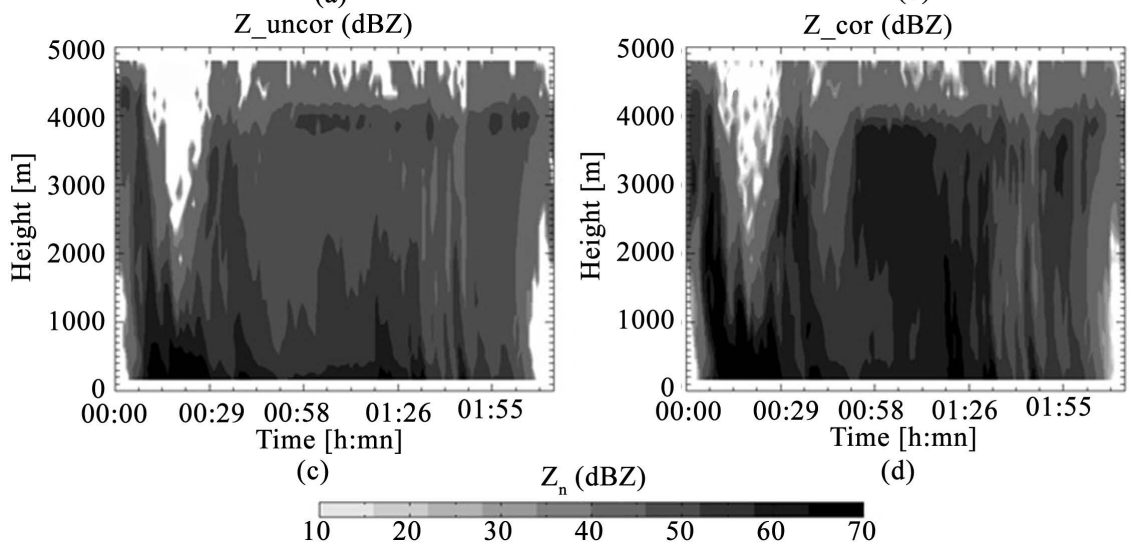

(d)

Figure 3. Fields of (a) measured, (b) corrected radar reflectivity factor from Xport radar (Top panels) and MRR plot of measured (c) and corrected (d) reflectivity factor $Z$ in dBZ (bottom panels) for the 02 August 2006 case in Nangatchori.

Figure 4 shows the comparison of the radar reflectivity factor measured by the MRR and the Xport radar before and after the attenuation and calibration corrections. The normalized bias (NB) displayed on the graphs has been improved from -0.23 to 0.04 , while the Nash-Sutcliffe criterion (Eff) has increased from -0.34 to 0.17 . Thus, overall, the reflectivity measurements, which were strongly underestimated, are slightly overestimated after the correction. However, 


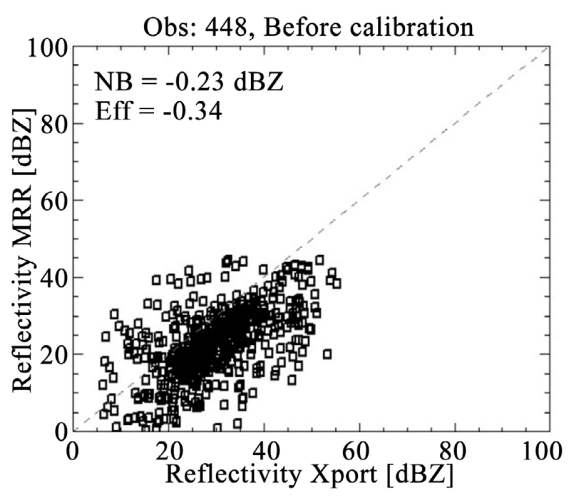

(a)

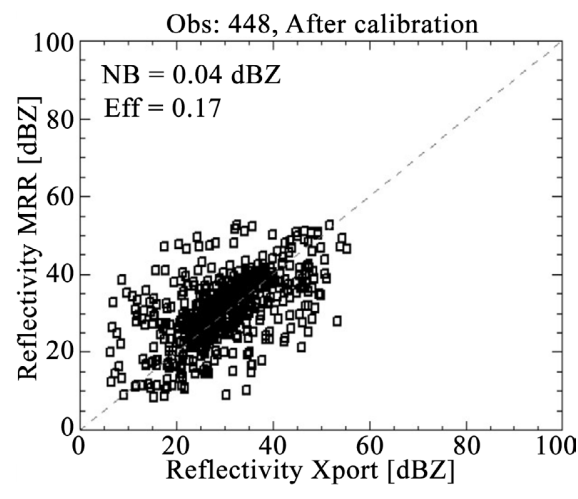

(b)

Figure 4. Scatter plots of Xport versus MRR reflectivity (a) before and (b) after correction of datasets. This scatter plots was produced with data collected by Xport and MRR in Nangatchori in 2006.

despite a very strong increase $(+0.51)$ in the Nash-Sutcliffe criterion, it remained low compared to the reference value of very good performance for this criterion, which is 1 . The reason for this is that even though the MRR and the Xport radar have a common measurement volume, due to the overlap of the two radar beams, there is a difference between the sampling volumes of the two radars. Specifically, the sampling volume of the Xport radar is much larger than that of the MRR. This difference between the sampling volumes of the two radars and the non-homogeneous filling of this volume could explain the relative weakness of the Nash-Sutcliffe criterion. However, we can see for reflectivity [20] [43] (dBZ) range, the corrections allowed a better dispersion of values around the first bisector. Alternatively, comparison of frequency histogram of MRR reflectivity values with the corresponding values from Xport was done (in Figure 5) with the same dataset those used to make Figure 4 . Figure 5 shows more coincident distributions after calibration and correction attenuation compared to results based on non-calibrated data from MRR. This is shown by a better agreement between probability density frequency curves of Xport and the MRR after data correction. Moreover, this is quantitatively confirmed by the close statistical scores (modal, mean and standard deviation values) shown in Figure 5. Basing to the fact that DSD is proportional to reflectivity, calibration correction also affects raindrop spectra and thus deduced rain rates.

As it can be observed in Figure 6, the results in term of rainfall show no significant differences between MRR and gauges measurements PDF after measurements corrected for attenuation and calibration issues applied to the MRR data. Correction algorithms result in a strong improvement of rainfall accumulation from MRR (21.6 mm before correction versus $211.9 \mathrm{~mm}$ ), which lead to a comparable value to rain gauges measurements $(187.9 \mathrm{~mm})$. Thus, despite some discrepancies, similarity between frequency distributions is obvious after MRR offset correction. After attenuation and calibration correction, the superimposition of the frequency distributions of rain rates retrieved by the MRR and rain 


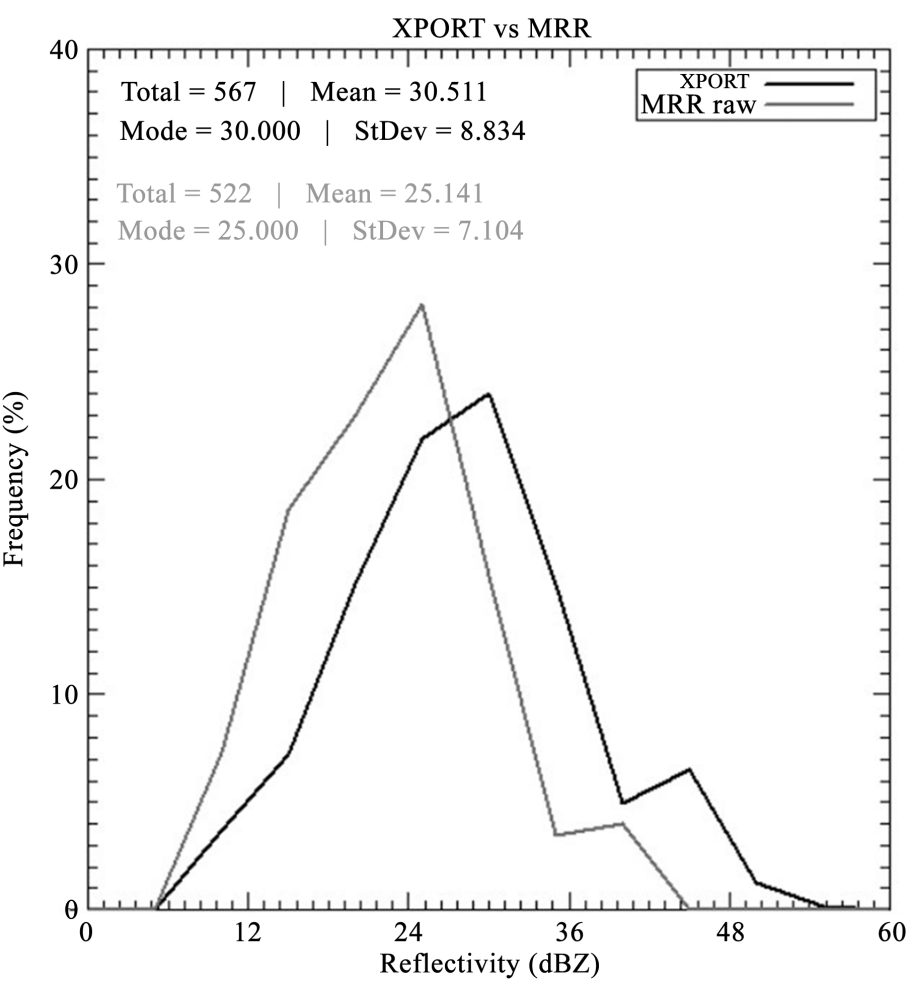

(a)

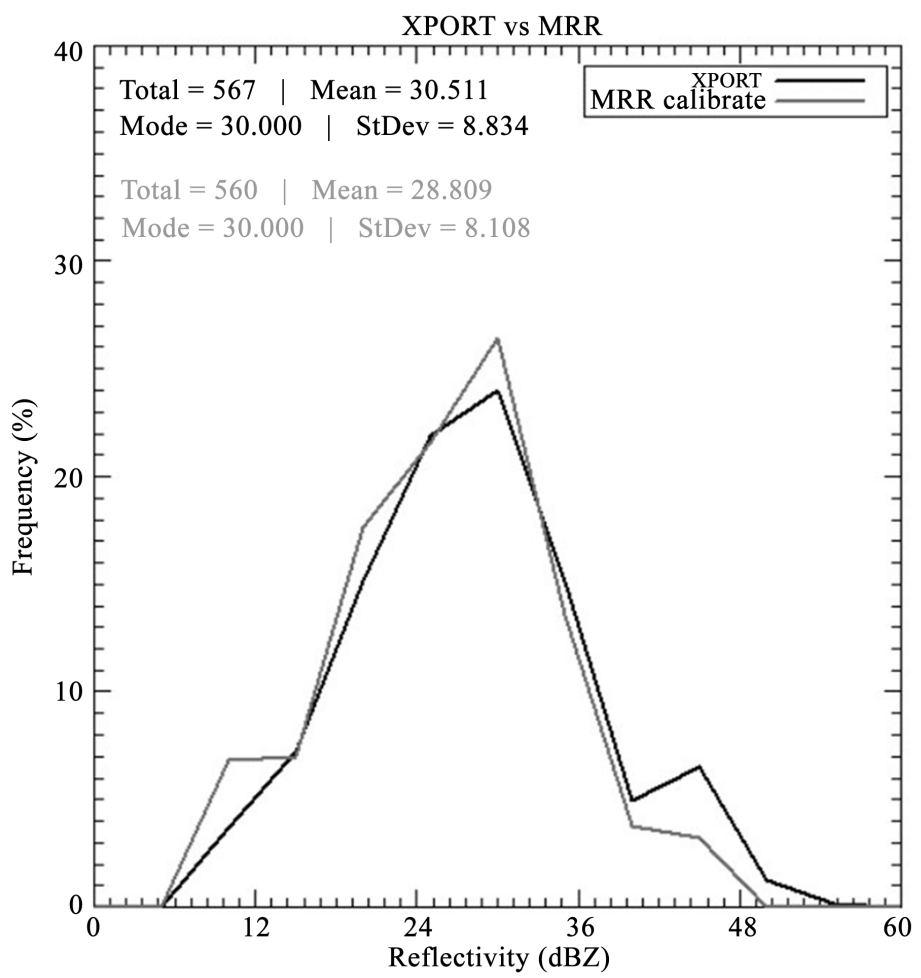

(b)

Figure 5. Comparison of frequency histogram of the MRR reflectivity values (a) before and (b) after attenuation and calibration correction vs reference determined reflectivity from Xport KDP-based attenuation correction. These distributions were carried out with data collected in Nangatchori during in 2006. 


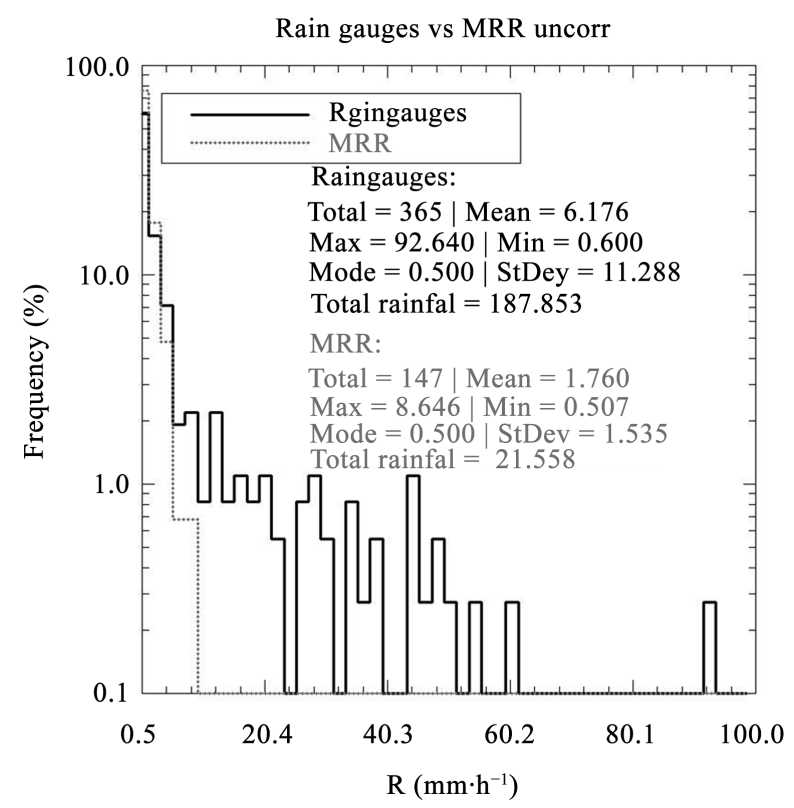

(a)

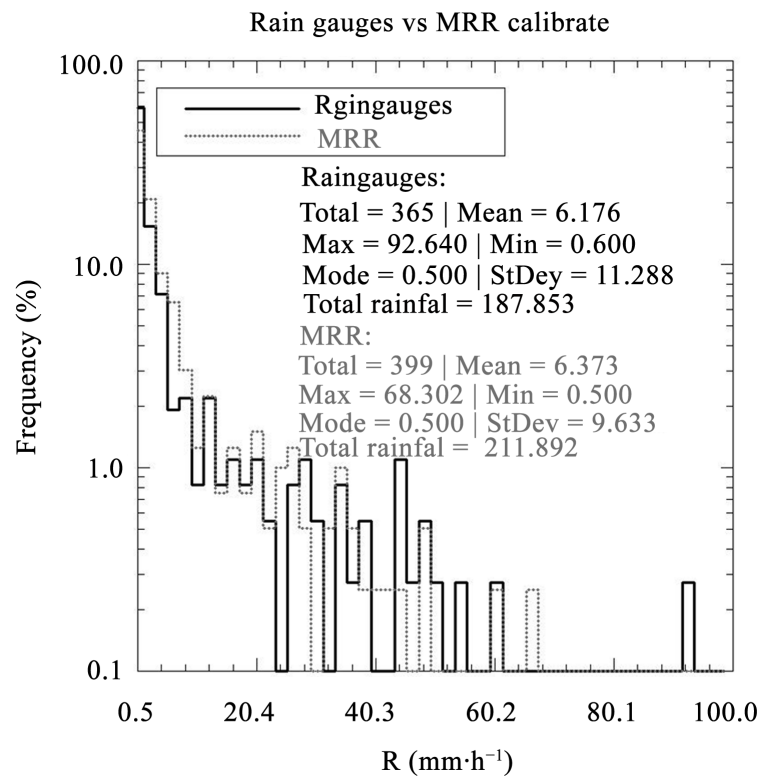

(b)

Figure 6. Frequency histogram of estimated rain rate from MRR measurements (a) before and (b) after data correction vs co-located Nangatchori gauge rainfall. These distributions were carried out with data collected in Nangatchori during in 2006.

rates measured by the rain gauges confirms the good agreement between the MRR and the rain gauges data.

Figure 7 shows the comparative evolution of the rates estimated and measured respectively by the MRR and rain gauges for three rain events (Figures 7(a)-(c)) and the dispersion curve (Figure 7(d)) for 32 rain events. For these three events, the MRR estimated the rainfall well and restituted relatively well the dynamics of the precipitation systems, as indicated by the performance criteria inscribed 


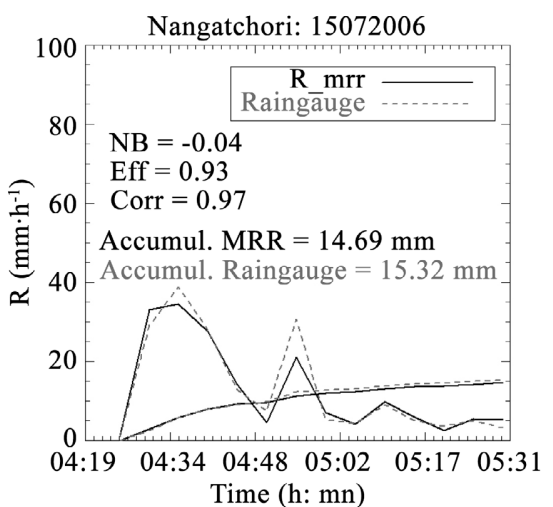

(a)

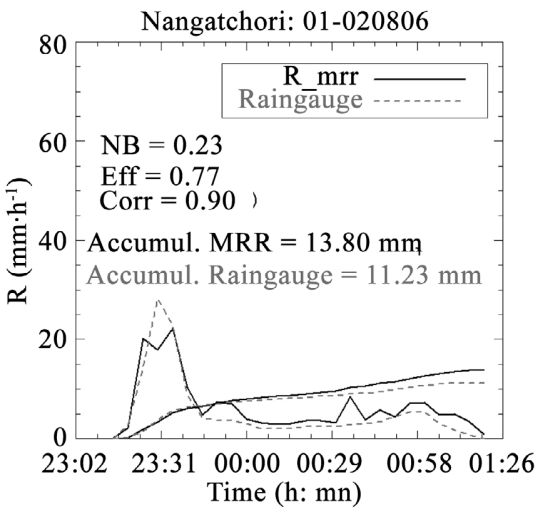

(c)

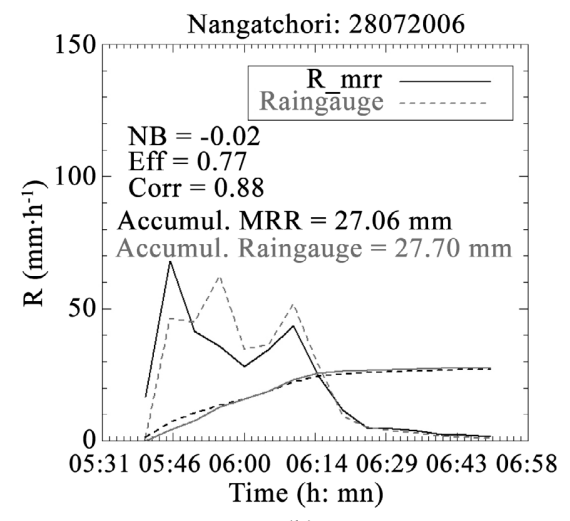

(b)

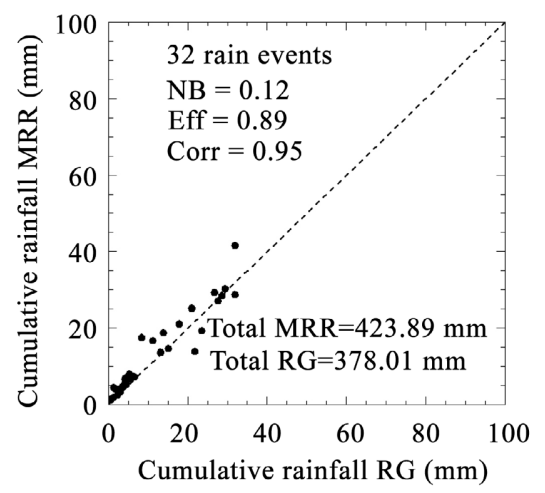

(d)

Figure 7. Time series of rainfall rates determined from MRR vs gauge direct measurements for three selected storm events in Nangatchori (a): 15/07/2006; (b): 28/07/2006; (c): 01-02/08/2006; and (d) comparison of event rainfall accumulations between gauge measurements and MRR estimates for 32 events in 2006 at Nangatchori MRR site.

on the different graphs. Indeed, for all the events presented, the correlation coefficients are higher than 0.8 , which indicates a good correlation between the rainfall estimated by the MRR and that measured by the rain gauges. The Nash-Sutcliffe coefficients are greater than 0.7 , indicating that the rainfall estimate by the MRR is of good quality. As for the normalized bias, its value attesting to the difference between the estimated and measured values is very low for the events of 15/07/2006 $(-0.04)$ and 28/07/2006 (-0.02) and much higher for the events of 01-02/08/2006 (0.23). In terms of the cumulative scatter plot (Figure $7(\mathrm{~d})$ ), the high correlation coefficient (0.95) indicates a strong correlation between the values estimated by the MRR and those provided by the rain gauges. The Nash-Sutcliffe coefficient value of 0.89 corroborates the performance of the correction algorithms applied to the MRR data which, at the scale of the rain event, reproduce the ground referenced accumulations. For the normalized bias, the value of 0.12 (12\%) globally indicates a slight overestimation of the event totals by the MRR, but this seems globally satisfactory.

Overall, there is an overestimation of rainfall by the MRR. This discrepancy between the rain estimated by the MRR and the in-situ observations could be explained by the fact that vertical wind effects were not taken into account in the 
processing of MRR data. Indeed, [44] and [45] found vertical wind to be a significant source of error, especially for heavy precipitation. In addition to the effect of vertical wind, there are the effects of turbulence, air density [36] and spectral folding [45] that were not considered in the processing chain. Also note the effects of sampling differences and the separation in altitude between the MRR ( $480 \mathrm{~m}$ is considered as the first valid gate) volume and the rain gauges (at ground) could also explain the discrepancy in measurements of these instruments. Comparisons between the corrected MRR data and Xport radar ones on the one hand and in-situ observational data provided by rain gauges on the other hand showed that the MRR is a good quality radar and can be used to estimate rainfall in a tropical environment such as West Africa rain medium.

\subsection{Microphysical Processes Classification}

The different microphysical processes that generated the rainfall at the Nangatchori site in 2006 during the AMMA campaign were discriminated against with the validated MRR data. Although the factors do not actually act alone, this analysis allows us to focus on dominant processes within convective systems. To this end, out of all the $N(D)$ drop distribution profiles explored, 18,810 profiles sampled every 10 s, i.e. 627 profiles averaged at 5-minute intervals, made it possible to unambiguously discriminate four dominant microphysical processes: coalescence, rupture, the combination of coalescence and rupture, and downdraft. Figure 8 shows a 5-minutes rainfall sequence, an illustration of these dominant microphysical processes, which reflect a variation in the concentration of drops from the tested altitudes to the reference altitude, on four example cases (or four 5-minutes rainfall sequence).

The criterion $N(D)_{\text {test }} / N(D)_{\text {ref }}>1$, reflecting a decrease in the concentration of raindrops, was observed for small drops while for medium or large drops, $N(D)_{\text {test }} / N(D)_{\text {ref }}<1$ (Figure 8(a)). This pattern suggests an aggregation of the small drops into either medium or large drops. Finally, the microphysical process illustrated in Figure 8(a) showed an overall decrease in the concentration of small drops and an increase in the concentration of medium and large drops between the tested and reference altitudes. All these changes in the shape of the DSD, illustrate a coalescence process that from a certain altitude onwards seems less efficient for the largest drop diameters $(3.5 \mathrm{~mm})$, as shown by the small differences in the concentration ratios for this range of diameters.

In Figure 8(b), the criterion $N(D)_{\text {test }} / N(D)_{\text {ref }}<1$, was found for small and medium drops, while we have $N(D)_{\text {test }} / N(D)_{\text {ref }}>1$ for large drops. These variations in $N(D)$ indicate a fragmentation of the large drops which led to an increase in the concentration of the small and medium drops. The changes in DSD shown in Figure 8(b) show an increase in the number of small and medium drops and a decrease in the number of large drops. This indicates that these changes in the DSD are indicative of a breakup process. It seems to have a greater effect on the number of drops with relatively larger vertical profile deviations for rain drops with a diameter less than $4 \mathrm{~mm}$. 


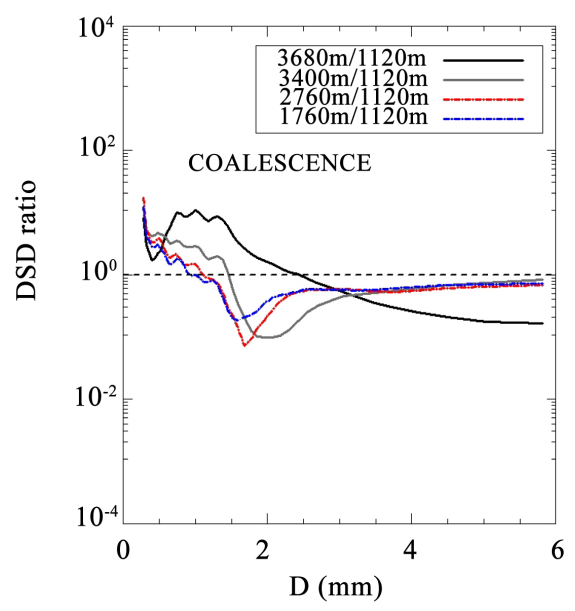

(a)

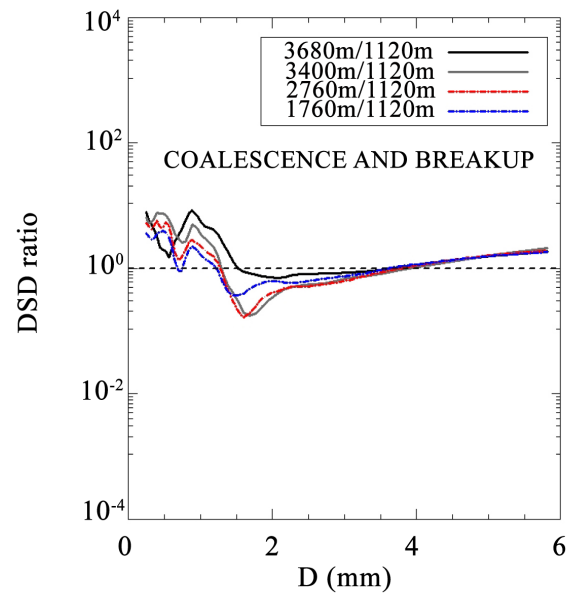

(c)

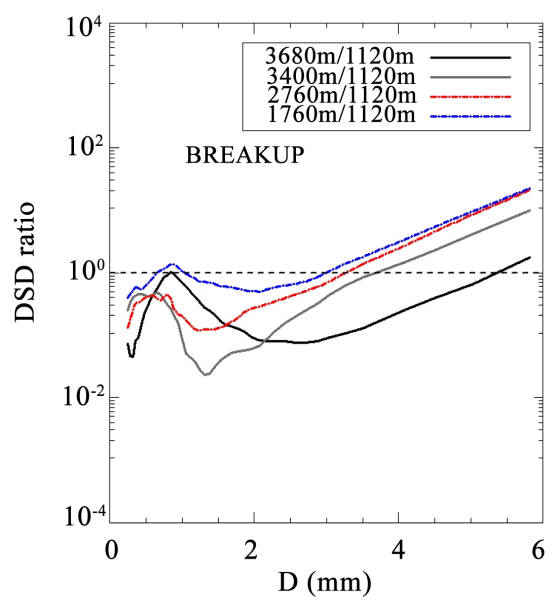

(b)

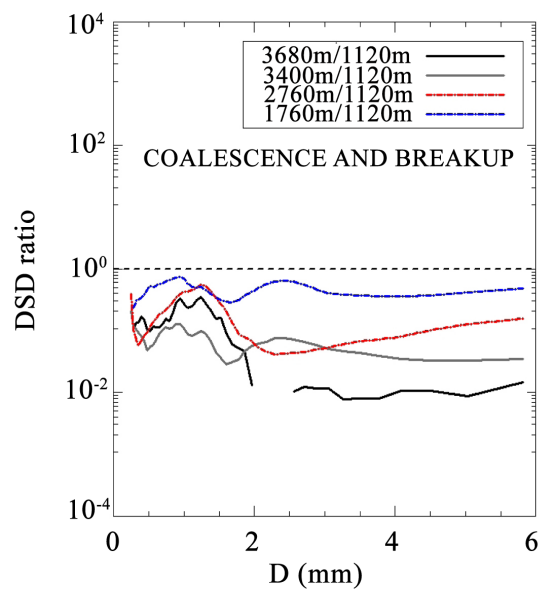

(d)

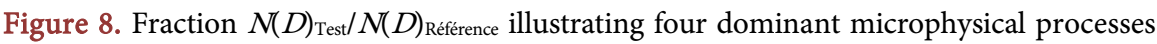
on the raindrop size distribution: (a) raindrop coalescence, (b) raindrop break-up, (c) coalescence and break-up acting simultaneously, and (d) downdraft effect from MRR observations. For the the sake of easy analysis, $N(D)_{\text {test }} / N(D)_{\text {ref }}>1$ (decrease) or $N(D)_{\text {test }} / N(D)_{\text {ref }}$ $<1$ (increase) in DSD at the referenced altitude $\sim 1 \mathrm{~km}$ with the respect to DSD at higher altitude should be considered at different diameter ranges such as small $(D<2 \mathrm{~mm})$, mid range $(2<D<4 \mathrm{~mm})$ and large $(D>4 \mathrm{~mm})$. On each figure, the four curves represent the variation of the DSD for 5 -minutes rainfall sequence.

The criterion $N(D)_{\text {test }} / N(D)_{\text {ref }}>1$ was observed for both small and large drops (see Figure $8(\mathrm{c})$ ). This indicates both a decrease in the concentration of small drops and large drops. Also in Figure 8(c), $N(D)_{\text {test }} / N(D)_{\text {ref }}<1$ was observed for medium size drops, suggesting an increase in the concentration of drops in this diameter range. Thus, the changes in DSD shown in Figure 8(c) induce an aggregation of small drops and a fragmentation of large drops. On the other hand, the aggregation of small drops and fragmentation of large drops led to an overall increase in the concentration of medium-size drops. Finally, the changes in DSD shown in Figure 8(c), led to a combined coalescence of small drops and break-up of large drops: indicating a process of combined coalescence and break-up. The 
combined action of coalescence and fragmentation would cause, compared to other microphysical processes, a relatively smaller variation with DSD altitude even for small raindrop diameters. According to [43], the combined action of coalescence and fragmentation of drops leads to equilibrium DSD as we see with drop concentrations independent of the altitudes considered for almost all drop diameters (concentration ratios close to 1 ).

In Figure 8(d), $N(D)_{\text {test }} / N(D)_{\text {ref }}<1$ was observed for all drop size ranges. These changes in the DSD indicate that the concentration of the number of drops regardless the diameter increases from the tested altitudes to the reference altitude as a result of the downdraft process. In Figure 8(d), an appearance of raindrops with diameters greater than $4.25 \mathrm{~mm}$ at altitudes below $3680 \mathrm{~m}$ is noted. This indicates a mechanism for the growth of drops during this downwind process in which particles undergo growth by accretion or collision-coalescence [46] with other particles among the small, intermediate and relatively large drops [12]. According to [46], collision efficiency increases with rainfall rate and is dependent on wind within the convective system.

Additional information characterizing the influences of microphysical processes can be determined through profiles of integrated DSD parameters such as the median volume diameter $\left(D_{0}\right)$, the concentration of the total number of drops per unit volume $\left(N_{T}\right)$ and their simultaneous consideration $\left(D_{0} / N_{T}\right)$ important in the adjustment of radar rainfall estimation algorithms [13]. Figure 9 shows the mean vertical profiles of these three parameters for all sequences identified by microphysical processes. Moreover, the mean evolution rates of $\mathrm{N}_{\mathrm{T}}$ as percentage are showed on Figure 9(b).

The median volumetric diameter $D_{0}$ increases from high to low altitudes for all four processes, but this increase is of variable amplitude depending on the microphysical processes considered. As regards the concentration of drops, the results show that $N_{T}$ decreases (with negative mean evolution rates) for all processes except the downwind process where it increases (positive mean evolution rate). However, the decrease of $N_{T}$ is stronger during the coalescence process than during the rupture process $(60.4 \%$ decrease rate for coalescence against $20.2 \%$ for the rupture process). In the present study, variations in DSD parameters during breakup processes seem to contradict the findings of [37]. Indeed, they showed that the breakup processes lead to an increase of $N_{T}$. However, based on the theoretical study of [46], it seems obvious that almost all of the breakup processes highlighted in our study are collision-breakup processes. These latter induce a decrease in $N_{T}$. In addition, [37] showed that $D_{0}$ increase and decrease respectively for coalescence and breakup processes. For the combination of coalescence and breakup processes, $D_{0}$ depends on the dominant process between coalescence and breakup. But, their study was theoretical, so ours used real data. In addition, [37] considered that each process acted alone, which is rarely the case. Thus, collision-breakup processes can give rise to collisions between drops and to evaporation processes which would influence $D_{0}$ differently. 


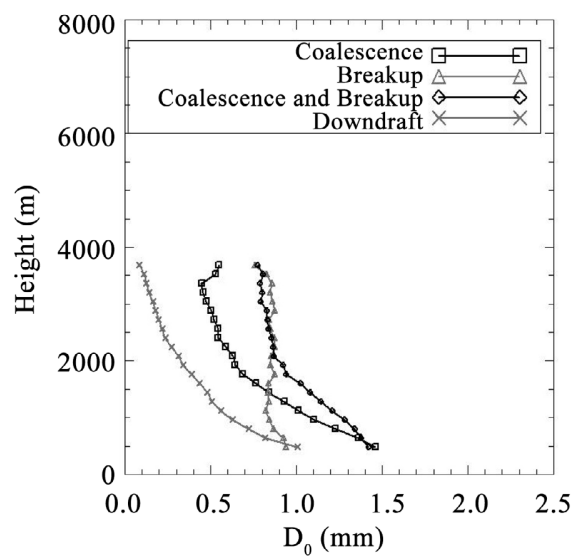

(a)

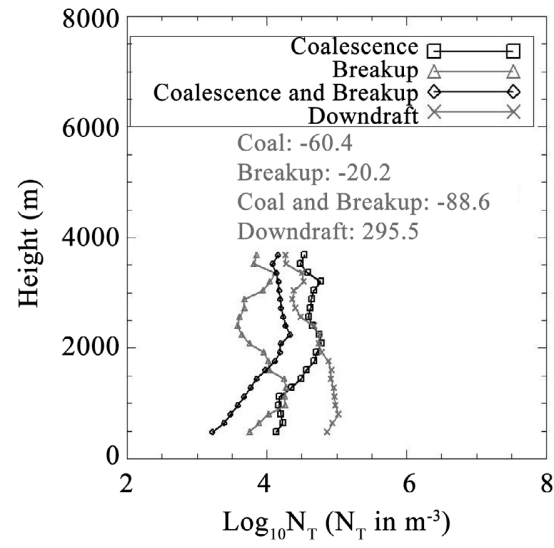

(b)

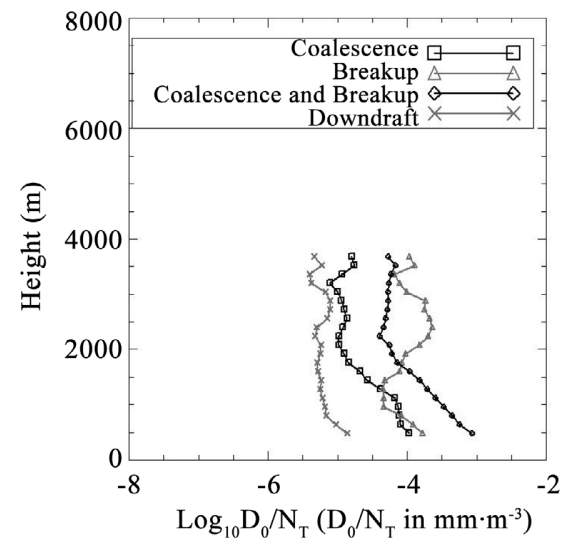

(c)

Figure 9. Mean vertical Profiles median diameter $D_{0}$ (a), Total raindrop size number $N_{T}$ (b) and their ratio $D_{0} / N_{T}$ (c) for all rainfall sequences identified as determining the processes of raindrop coalescence, break-up, coalescence and break-up acting simultaneously, and downdraft.

On average, raindrop fragmentation is a process in which the mode of variability of the DSD is controlled by variation in the total number of raindrops, with little variation in the median volume diameter. The evolution of $D_{0} / N_{T}$ is then inferred from the inverse of that of $N_{T}$ to within a multiplicative factor. A similar behavior is attributed to the downdraft process for which the dependence on $N_{T}$ is rather low for lowest values of $D_{0}\left(D_{0}<1 \mathrm{~mm}\right)$ despite a relatively large variation with altitude. On the other hand, the coalescence and coalescence-breakup processes are characterized by the combined variation in drop size $\left(D_{0}\right)$ and $N_{T}$. Indeed, the variations of $D_{0} / N_{T}$ in the DSDs of these two processes follow those of $D_{0}$ while having curves at the opposite paces to that of $N_{T}$. The main difference between these two processes would be related to the differences in the total concentration profiles of the large drops $(D \geq 4 \mathrm{~mm})$ when they occur separately (Figure 10). Thus, since microphysical processes do not necessarily act alone in convective systems, whether or not a process is effective in the formation of such large droplets would determine the acuity of the $D_{0} / N_{T}$ variation mode of DSD for these two processes. 


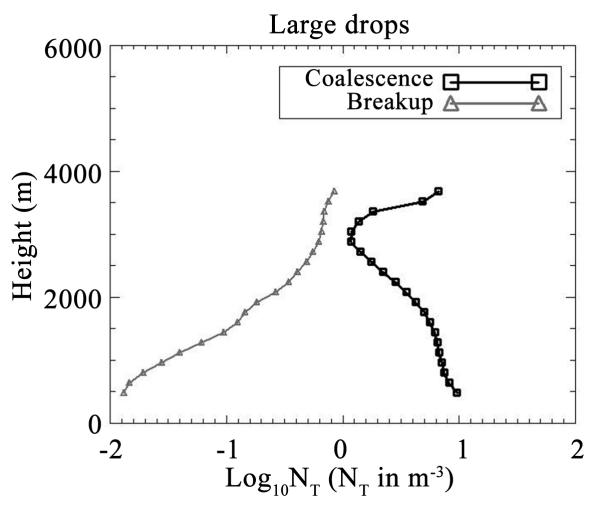

Figure 10. Mean vertical profiles (5-min rainfall sequence) of the total raindrop size number $N_{T}$ for large raindrop diameter $(D \geq 4 \mathrm{~mm})$ for coalescence and break-up.

Based on the 627 DSD profiles sampled in 5-min steps (about 3135 1-min spectra) of discriminated raindrop size distributions, there are (415 1-min spectra) for coalescence, (550 1-min spectra) for breakup, (1610 1-min spectra) for the combination of coalescence and breakup, and $560 \mathrm{~min}$ for the downdraft process. These different microphysical processes then represent 13.3\% (coalescence), 17.6\% (breakup), 51.4\% (combination of coalescence and breakup) and 17.86\% (downdraft) of the data set used. Thus, the processes of coalescence and rupture or the combination of these two represented $82.14 \%$ of the processes highlighted with the method that was used. This confirms the conclusions of [47] that the shape of the raindrop size distribution is essentially controlled by coalescence and rupture processes (in which it includes collision and collection processes). However, it should be noted that the method used in the present study did not reveal microphysical processes such as the processes of evaporation, collection, upwind and sorting of drops according to their size.

\subsection{Variability of $Z-R$ Relations in Connection with Microphysical Processes and DSD Parameters}

The DSD parameters and the $A$ and $b$ coefficients of the $Z-R$ relations were determined for each dominant microphysical process. In this section, the connection between the microphysical processes and the DSD parameters was investigated. In addition, the variability of $Z-R$ relationships as a function of dominant microphysical processes and DSD integrated parameters was also analyzed.

Figure 11 shows the vertical profile of the $A$ and $b$ coefficients of the $Z-R$ relationships for the four microphysical processes derived from the MRR dataset used in this study. A variation in the $A$ factor and $b$ exponent within each microphysical process and between microphysical processes was observed as a function of altitude. A clear difference in coefficient values of $Z-R$ relations between microphysical processes is the main feature although at certain altitudes there are similar values between coalescence and coalescence-break at one hand and breakup and downdraft processes. Obviously, it was found that

$A_{C}>A_{C B}>A_{B}>A_{D}$ (see Figure 11(a)), where $A_{C}, A_{C B}, A_{B}$ and $A_{D}$ represent 


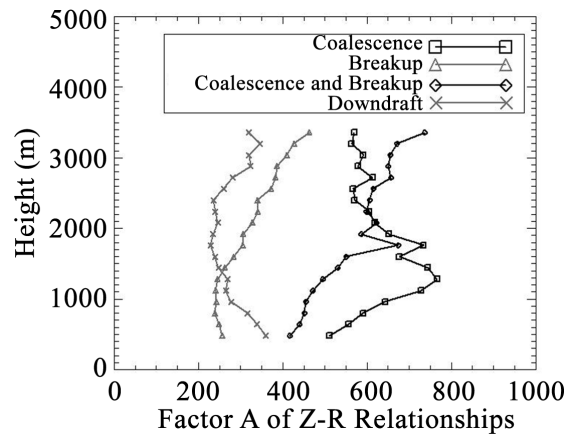

(a)

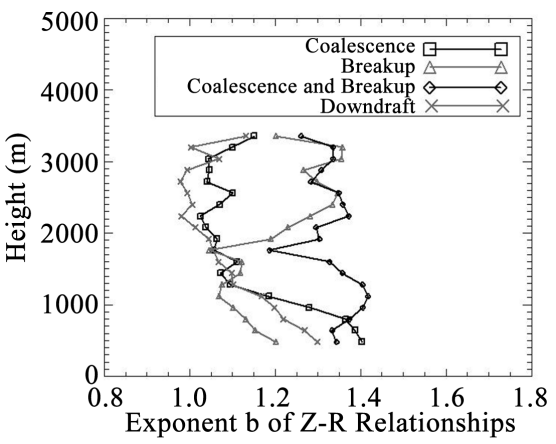

(b)

Figure 11. Vertical profile of $Z$ - $R$ relationship (a) pre-factor $A$, (b) exponent $b$, for different microphysical processes.

the pre-factor A for the coalescence, the combined coalescence and breakup, the breakup and downdraft processes respectively. According [12], large coefficient could be attributed to the presence of large raindrops produced by coalescence and collision breakup (size or mixed controlled processes) which are also associated with small exponents, whereas the small coefficients associated with large exponents are induced by the presence of large number density (number controlled processes) in the small drop diameter range. In contrast, based on results from Figure 11, for altitude lesser than $1300 \mathrm{~m}$, large (small) exponent are linked to large (small) coefficient and differences between microphysical processes become more prominent and clear, unlike the unprecedented and sometimes ambiguous situations of variability of exponent $b$ at higher altitudes. Given the highly uncertain evolution of exponent $b$ from one microphysical process to another and within a microphysical process (Figure 11(b)) it seems obvious analyzing $Z-R$ variability by taking into account simultaneously the size and number of raindrops [13]. Such an approach derives its success from the fact that in reality the processes do not act alone. Indeed, very often, we can be in the presence of a conjunction of number and/or size controlled processes, according to the range of diameter classes.

As illustrated by Figure 9(b), Figure 11(a) and Figure 11(b), the higher value of $D_{0}$ (coalescence process), corresponds to the higher value of pre-factor $A$ (as the exponent $\mathrm{b}$ ) while the lower value of $D_{0}$ (process of rupture) corresponds to the lower of the factor $A$ (like the exponent $\mathrm{b}$ ). Likewise, the higher value of prefactor $A$ (as the exponent b) corresponds to the higher value of $N_{T}$ (Figure 9(b) and Figure 11(a) and Figure 11(b)). The $D_{0} / N_{T}$ ratio, relating to the coalescence process is lower than that relating to the breakup process (see Figure $9(\mathrm{c})$ ). In addition, the pre-factor $\mathrm{A}$ of the coalescence process is higher than that of the rupture process (see Figure 11(a)). Thus, from comparison with $D_{0} / N_{T}$, two distinct main cases are evolved:

- For coalescence and coalescence-breakup processes

If $\left(D_{0} / N_{T}\right)_{P 1}<\left(D_{0} / N_{T}\right)_{P 2}$ then $A_{P 1}>A_{P 2}$ and $b_{P 1}<b_{P 2}$ pointing to an inverse evolution of $D_{0} / N_{T}$ and coefficient, unlike similar evolution with exponent. 
- For breakup and downdraft processes

If $\left(D_{0} / N_{T}\right)_{P 1}>\left(D_{0} / N_{T}\right)_{P 2}$ then $A_{P 1}>A_{P 2}$ and $b_{P 1}>b_{P 2}$ with, $P 1$ and $P 2$ are two distinct rainy sequences.

Thus, both value of exponent $\mathrm{b}$ and pre-factor A being highly depending on DSD integrated parameters, therefore it strongly suggests the need to determine coefficients of $Z-R$ algorithm by involving microphysics assumption that govern the DSD.

\subsection{Rainfall Retrieval Algorithms Evaluation}

To reduce bias in rainfall estimates compared to ground reference measurements, estimators based on MRR measurements are developed with data as close to the ground as possible. For this purpose, the reflectivity $Z$ and the rain rate $R$ of the third gate of the MRR which is the first valid gate are used. With regard to the algorithms taking into account microphysical processes, the coefficients of the unique relationships obtained are the averages of the coefficients determined per process weighted by the percentages of occurrence of the processes analyzed in the rainfall sequences observed by the MRR. Thus, the following algorithms were obtained:

- Without taking into account the dominant microphysical processes (non-linear least squares adjustment): $Z=208 R^{1.55}$;

- With consideration of dominant microphysical processes (non-linear least squares adjustment): $Z=253 R^{1.66}$.

As a comparison with the recent $Z-R$ relationships applied to the same Xport data we indicate three algorithms from the literature, including the $Z=655 R^{1.29}$ algorithm of [19] determined by fitting radar-rain gauge data from the intensive AMMA 2006-2007 campaign. The second ( $Z=460 R^{1.36}$ ) was obtained by adjusting $Z-R$ estimators based on radar and rain gauge comparisons, from $Z-R$ estimators previously established for West Africa [18] [19] [21]. It is a fitting method suggested by [48] which consists in searching for an optimal relationship by traversing the space of the values of the coefficients $A$ and $b$ in ranges drawn from algorithms in the literature on AMMA observations [49]. The so-best algorithm called in the following "fitted estimators" satisfies a set of criteria contained in a cost function integrating a good distribution of rain rates scatterplot estimated by this relation around the first bisector by giving more weight to high values, a good agreement between the rain rate probability distribution frequency (PDFs) estimated and measured by the rain gauge network, a good agreement of the class contributions to the total accumulation (in other words, evaluating the contributions of class rainfall PDFs weighted by the average rain rate of the class). The third algorithm ( $Z=218 R^{1.62}$ ) also derived from [19] is obtained by simulating the disdrometric dataset of the AMMA campaign [18] [21] by making the assumptions of Andsager's raindrop shape model [35] and a temperature of $25^{\circ} \mathrm{C}$.

Given that the determination of $Z-R$ coefficients is sensitive to the analytical methods used [39], and that the performance of our estimators is evaluated by 
comparison with optimized algorithms in the literature, we considered only optimized $Z-R$ algorithms for rainfall estimation. Thus, the results of linear least squares $Z$ - $R$ estimators are not displayed.

For the evaluation of the algorithms, the horizontal reflectivities measured by the Xport radar in 2006 during the intensive AMMA campaign in Benin are used. The different rainfall estimates are then compared with ground measurements provided by rain gauges located within $50 \mathrm{~km}$ around Xport radar.

Figure 12 shows scatterplots of 1 ) MRR-based optimized $Z-R$ without microphysics' processes, 2) optimized $Z-R$ accounting microphysical processes, 3) radar-rain gauges fitting $Z-R, 4) Z-R$ estimators fitting based on those from AMMA's literature and e) DSD-based simulated $Z$ - $R$ against 5-minutes time steps gauges accumulations. It indicates that estimators derived from vertical pointing radar (MRR) measurements perform better on the fine time scale than those derived from radar-rain gauges observations. In terms of coefficient of efficiency (Nash criterion), the algorithms dependent on DSDs from the MRR are 1.5 to 2.5 times better than the others. This highlights the interest of establishing these algorithms from measurements from the same instrument to remove the biases introduced by the sampling difference between the measuring devices. Indeed, the algorithm obtained by simulation from the disdrometer data alone also has performances relatively close to the MRR algorithms. In particular, the algorithm based on microphysical processes gives the lowest normalized standard error among all the algorithms and the associated Nash coefficient (0.708) in terms of bulk statistics (all rain rates are considered) is 1.5 times higher than that of the algorithm derived from the MRR data abstracting from microphysics. Thus, with this evaluation, it emerges that knowledge of the DSD is decisive for a more suitable $Z-R$ relationship for rainfall estimation. In addition, the best estimates are obtained by including the dominant microphysical processes underlying the formation of the DSD, particularly for estimates with a time step of 5 minutes. At rainfall event scale (Figure 13), the climatologically relationship derived from simulations based on all DSDs of the AMMA intensive campaigns has estimates comparable to those of the algorithm that suggest taking into account the dominant microphysical processes. The similarity of their Nash and NRMSE values is striking (NRMSE $=0.25 / \mathrm{Eff}=0.84$ for algorithm taking into account the microphysical processes versus NRMSE $=0.22 / \mathrm{Eff}=0.86$ for $Z$ - $R$ disdrometer-derived estimator). This suggests that, at rainfall event scale, all the processes through the DSD measured at the ground is a reliable alternative for the determination of adapted $Z-R$ algorithms reducing biases in the rainfall estimates. The closeness of the results of these two algorithms shows the interest of taking into account microphysical processes in their determination, giving that estimator based on DSDs dataset of the AMMA campaign includes implicitly various events in which different microphysical processes are combined. However, using algorithms derived from the ground-based DSD, we should keep in mind that the increased presence of large diameter raindrops at high altitudes where the radar 


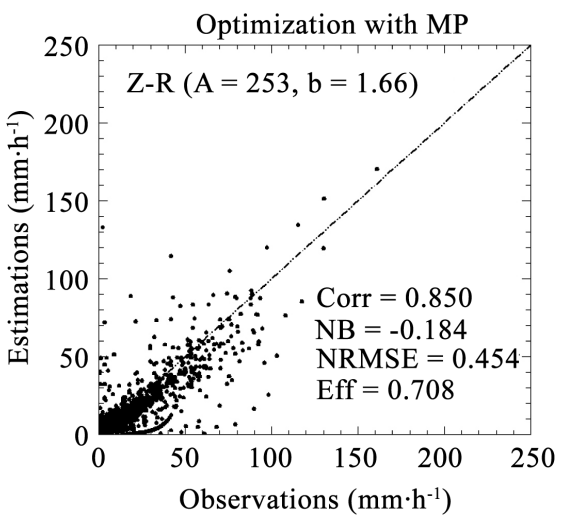

(a)

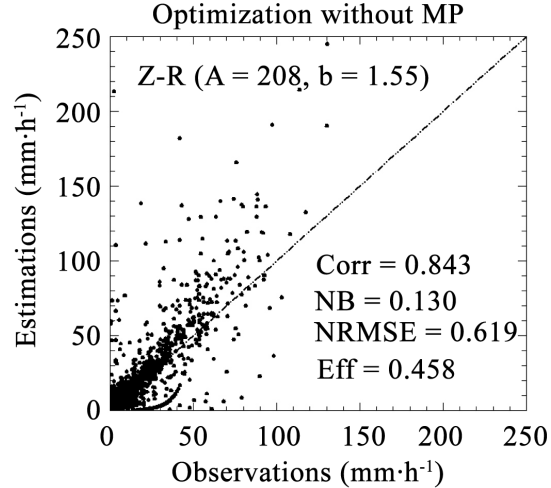

(b)

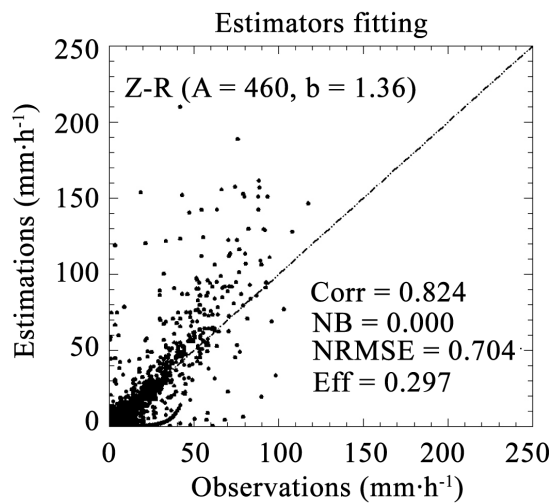

(d)

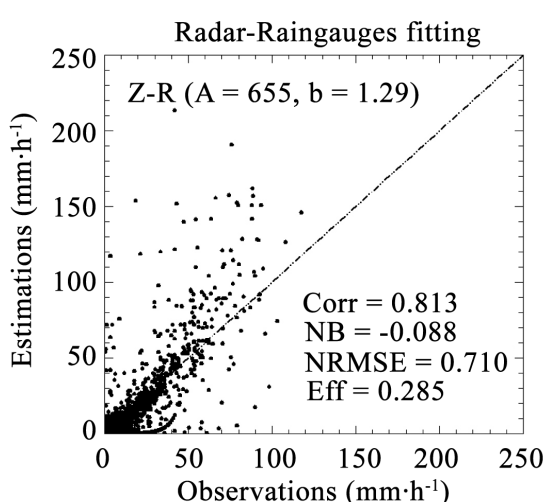

(c)

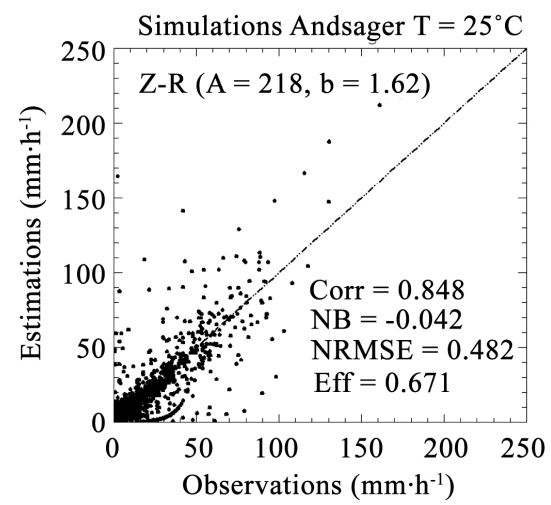

(e)

Figure 12. Scatter plots of rainfall rates retrieved by five $Z-R$ algorithms against rainfall rates measured by rain gauges: (a) $Z=208 R^{1.55}$ (non-linear regression without taking into account microphysical processes called "Optimization without MP"), (b) $Z=253 R^{1.66}$ (non-linear regression with microphysical processes called “Optimization with MP”), (c) $Z=655 R^{1.29}$ (fitting radar-rain gauge datasets: "Radar-rain gauges fitting”), (d):

$Z=460 R^{1.36} \quad$ (adjusting $Z-R$ estimators based on radar and rain gauge comparisons: "Estimators fitting"), (e) $Z=218 R^{1.62}$ (Algorithm derived from T-matrix simulations based on DSDs gathered during AMMA campaigns: "Simulations Andsager $T=25^{\circ} \mathrm{C}$ ”).

measurements are done would limit the quality of these estimators, since $Z-R$ is sensitive to the presence of large diameter raindrops [1] [50]. Such exceptional diameters are generally unobserved in ground-based DSDs since raindrop 


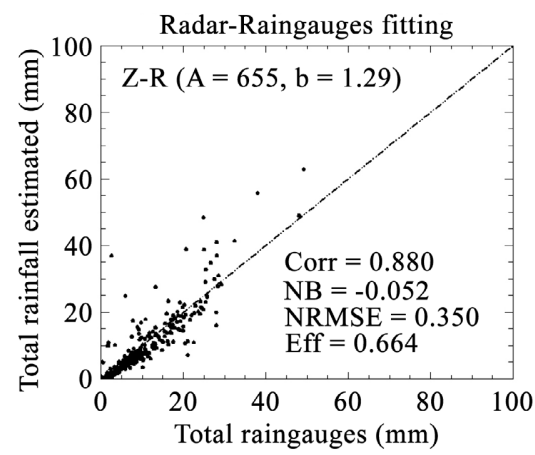

(a)

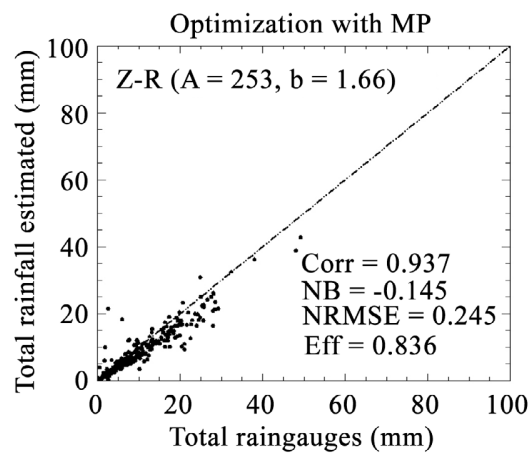

(c)

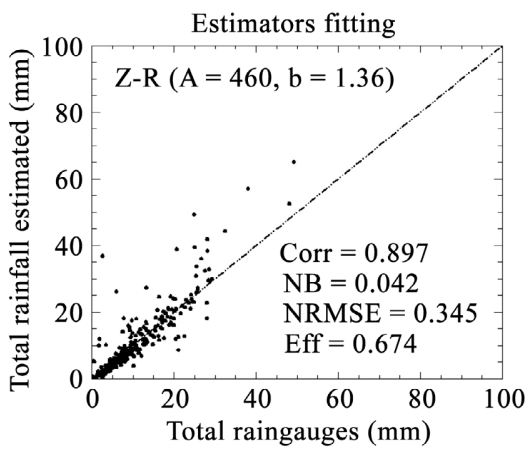

(b)

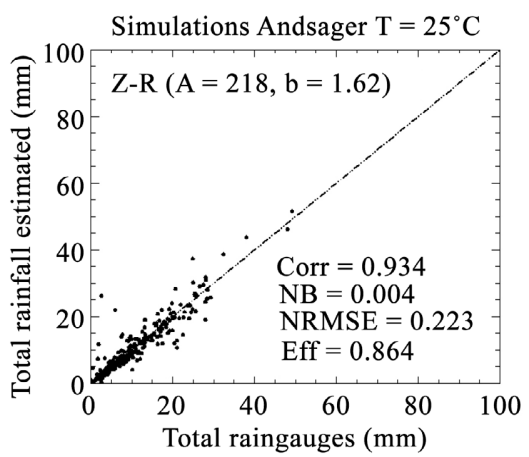

(d)

Figure 13. Scatter plots of rainfall event accumulation retrieved from Xport radar reflectivity by employing four most performing $Z-R$ algorithms ("Radar-rain gauges fitting", "Estimators fitting", “Optimization with MP", "Simulations Andsager $T=25^{\circ} \mathrm{C}$ ”) against rainfall measured by rain gauges.

breakup processes reduce their occurrence. Since rainfall events are monitored by radar at finer time steps, an algorithm that integrates the highly variable microphysical processes during the life cycle of the convective systems [51] and from one rainfall event to another is highly recommended [1]. In comparison with the work of [19], such an algorithm would seem to be as efficient as polarimetric estimators even without setting a threshold on the reference intensities.

Overall, with this $Z-R$ algorithm taking into account microphysical processes, an important step is done in the way to reduce errors in rainfall estimation introduced by the variability of microphysical processes, as suggested by [1]. As a comparison [52], with the idea of including the consideration of microphysical processes in rainfall estimation algorithms [53], have designed an algorithm (named SCOP-ME) that simultaneously includes integrated DSD parameters associated with drop size (the median volume diameter $D_{0}$ ) and their number (the interception parameter $N_{W}$ ). These parameters $N_{W}$ and $D_{0}$ are estimated from the polarimetric radar variables. The evaluation of this algorithm, in terms of rainfall estimates, gave for different integration times (15/30/60 minutes) Nash coefficients $(0.82 / 0.79 / 0.83$ respectively for corresponding time steps) of the same order as the $Z-R$ algorithm based on a more direct consideration of the microphysical processes developed in our work ( 0.84 at rainfall event scale and 0.71 at the $5 \mathrm{~min}$ time step). In terms of NRMSE, SCOP-ME (0.63; 0.59; 0.51 for 15, 30, 60 minutes 
integration time respectively) remains less efficient than $Z-R$ adjusted by the discrimination of the microphysical processes $(0.25$ and 0.45 at the rainfall event scale and at the $5 \mathrm{~min}$ time step, respectively). Certainly, the NRMSE high values of SCOP-ME rainfall estimates depends on the performance of the $N_{w}$ and $D_{0}$ retrieval algorithms as implicitly recognized by its authors [52].

\section{Summary and Conclusions}

In this study, the devices (a Micro Rain Radar, a polarimetric X-band horizontal scanning radar, a dense network of rain gauges) for radar experiment during the AMMA radar campaign in 2006 that operated in Northern Benin for monitoring the rainfall systems in the region and estimating rainfall provided an appropriate framework for evaluating a $Z-R$ algorithm including the microphysical processes within the rainstorms monitored. Such a framework offered the opportunity to exploit for the first time the MRR data gathered during AMMA by taking advantage of the co-location of a polarimetric X-band radar to correct the rain attenuation and calibration issues that hampered the expectation of having such low cost micro radar. The main conclusions drawn from this study are summarized as follows:

- The normalized DSD variations showed a clear relation between raindrop concentration, DSD integrated parameters and coalescence, coalescence-breakup, breakup, and downdraft processes. Micro rain radar DSD-derived Z-R relations according the various processes above mentioned are found to be different from a process to another according that size, number or mix controlled modes are dominant within rainstorms.

- The unique adjusted $Z-R$ algorithm coefficients including various microphysical processes are derived as the averages of the coefficients determined per dominant process weighted by the percentages of occurrence of these processes. In this case, nonlinear regression is established to determine optimal coefficients comparable to existing optimized $Z-R$ relations.

- The performance of microphysical processes induced $Z-R$ algorithm was compared against three other optimized $Z-R$ in literature based on AMMA dataset. Bulk statistics comparison exhibits that $Z-R$ algorithm involving microphysical processes outperforms the other in terms of Nash coefficient and normalized standard error.

- The impact of taking into account the dominant microphysical processes in the $Z-R$ quantitative rainfall estimation is also evaluated by comparison to rainfall rate estimates with SCOP-ME, a polarimetric algorithm involving rain microphysics retrievals [52]. In terms of error and efficiency statistics, sufficiently similar results are found.

Although the $Z-R$ relationship calibration based on the microphysical processes led to progress in radar QPE, future studies focusing on the combination of microphysical processes discrimination by precipitation type (light or heavy rain) and derived subsequent $Z$ - $R$ optimized estimators, not dealt with in the present 
study, could certainly improve quantitative precipitation estimation. Furthermore, since rainfall events are monitored by radar at finer time steps, an algorithm that integrates the highly variable microphysical processes during the life cycle of the convective systems and from one rainfall event to another is highly recommended.

\section{Acknowledgements}

This research was conducted under the auspices of AMMA. Based on the French initiative, AMMA was built by an international scientific group. A large number of agencies, especially from France, the United Kingdom, the USA and Africa, currently fund it. It has been the beneficiary of a major financial contribution from the European Community's Sixth Framework Research Program. Detailed information on scientific coordination and funding is available on the AMMA international website: http://www.amma-international.org.

\section{Conflicts of Interest}

The authors declare no conflicts of interest regarding the publication of this paper.

\section{References}

[1] Lee, G.W. and Zawadzki, I. (2005) Variability of Drop Size Distributions: Time-Scale Dependence of the Variability and Its Effects on Rain Estimation. Journal of Applied Meteorology and Climatology, 44, 241-255. https://doi.org/10.1175/JAM2183.1

[2] Atlas, D., Ulbrich, C.W., Marks, J.F.D., Amitai, E. and Williams, C.R. (1999) Systematic Variation of Drop Size and Radar-Rainfall Relations. Journal of Geophysical Research, 104, 6155-6169. https://doi.org/10.1029/1998JD200098

[3] Sharma, S., Konwar, M., Sarma, D.K., Kalapureddy, C.R. and Jain, A.R. (2009) Characteristics of Rain Integral Parameters during Tropical Convective, Transition, and Stratiform Rain at Gadanki and Its Application in Rain Retrieval. Journal of Applied Meteorology and Climatology, 48, 1245-1266. https://doi.org/10.1175/2008JAMC1948.1

[4] Tokay, A. and Short, D.A. (1996) Evidence from Tropical Raindrop Spectra of the Origin of Rain from Stratiform versus Convective Clouds. Journal of Applied Meteorology and Climatology, 35, 355-371. https://doi.org/10.1175/1520-0450(1996)035<0355:EFTRSO >2.0.CO;2

[5] Yuter, S.E. and Houze, R.A. (1997) Measurements of Raindrop Size Distribution over the Pacific Warm Pool and Implementations for Z-R Relations. Journal of Applied Meteorology and Climatology, 36, 847-867. https://doi.org/10.1175/1520-0450(1997)036<0847:MORSDO >2.0.CO;2

[6] Rao, T.N., Rao, D.N, Mohan, K. and Raghavan, S. (2001) Classification of Tropical Precipitating Systems and Associated Z-R Relationships. Journal of Geophysical Research, 106, 17699-17711. https://doi.org/10.1029/2000JD900836

[7] Maki, M., Keenan, T.D., Sasaki Y. and Nakamura, K. (2001) Characteristics of the Raindrop Size Distribution in Tropical Continental Squall Lines Observed in Darwin, Australia. Journal of Applied Meteorology and Climatology, 40, 1393-1412. https://doi.org/10.1175/1520-0450(2001)040<1393:COTRSD>2.0.CO;2 
[8] Licznar, P. and Krajewski, W.F. (2016) Precipitation Type Specific Radar Reflectivity-Rain Rate Relationships for Warsaw, Poland. Acta Geophysica, 64, 1840-1857. https://doi.org/10.1515/acgeo-2016-0071

[9] Kozu, T., Reddy, K.K., Mori, M., Thurai, M., Ong, J.T., Rao, D.N. and Shimomai, T. (2006) Seasonal and Diurnal Variations of Raindrop Size Distribution in Asian Monsoon Region. Journal of the Meteorological Society of Japan, 84, 195-209. https://doi.org/10.2151/jmsj.84A.195

[10] Seela, B.K., Janapati, J., Lin, P.-L., Wang, P.K. and Lee, M.-T. (2018) Raindrop Size Distribution Characteristics of Summer and Winter Season Rainfall over North Taiwan. Journal of Geophysical Research: Atmospheres, 123, 11602-11624. https://doi.org/10.1029/2018JD028307

[11] Tenorio, R.S., Moraes, M.C.S. and Sauvageot, H. (2012) Raindrop Size Distribution Radar Parameters in Coastal Tropical Rain Systems of Northeastern Brazil. Journal of Applied Meteorology and Climatology, 51, 1960-1970.

https://doi.org/10.1175/JAMC-D-11-0121.1

[12] Konwar, M., Maheskumar, R.S., Das, S.K. and Morwal, S.B. (2012) Nature of Light Rain during Presence and Absence Bright Band. Journal of Earth System Science, 121, 947-961. https://doi.org/10.1007/s12040-012-0202-x

[13] Ochou, A.D., Zahiri, E.-P., Bamba, B. and Koffi, M. (2011) Understanding the Variability of Z-R Relationships Caused by Natural Variations in Raindrop Size Distributions (DSD): Implication of Drop Size and Number. Atmospheric and Climate Sciences, 1, 147-164. https://doi.org/10.4236/acs.2011.13017

[14] Hasan, M.M., Sharma, A., Johnson, F., Mariethoz, G. and Seed, A. (2016) Improving Radar Rainfall Estimation by Merging Point Rainfall Measurements within a Model Combination Framework. Advances in Water Resources, 97, 205-218. https://doi.org/10.1016/j.advwatres.2016.09.011

[15] Steiner, M., Smith, J.A. and Uijlenhoet, R. (2004) A Microphysical Interpretation of Radar Reflectivity-Rain Rate Relationships. Journal of the Atmospheric Sciences, 61, 1114-1131. https://doi.org/10.1175/1520-0469(2004)061<1114:AMIORR>2.0.CO;2

[16] Konwar, M., Das, S.K, Deshpande, S.M., Chakravarty, K. and Goswami, B.N. (2014) Microphysics of Clouds and Rain over the Western Ghat. Journal of Geophysical Research: Atmospheres, 119, 6140-6159. https://doi.org/10.1002/2014JD021606

[17] Xie, X., Evaristo, R., Simmer, C., Handwerker, J. and Trömel, S. (2016) Precipitation and Microphysical Processes Observed by Three Polarimetric X-Band Radars and Ground-Based Instrumentation during HOPE. Atmospheric Chemastry and Physics, 16, 7105-7116. https://doi.org/10.5194/acp-16-7105-2016

[18] Gosset, M., Zahiri, E-P. and Moumouni, S. (2010) Rain Drop Size Distribution Variability and Impact on X-Band Polarimetric Radar Retrieval: Results from the AMMA Campaign in Benin. Royal Meteorological Society (RMetS), 136, 243-256. https://doi.org/10.1002/qj.556

[19] Koffi, A.K., Gosset, M., Zahiri, E.P., Ochou, A.D., Kacou, M., Cazenave, F. and Assamoi, P. (2014) Evaluation of X-Band Polarimetric Radar Estimation of Rainfall and Rain Drop Size Distribution Parameters in West Africa. Atmospheric Research, 143, 438-461. https://doi.org/10.1016/j.atmosres.2014.03.009

[20] Depraetere, C., Gosset, M., Ploix, S. and Laurent, H. (2009) The Organization and Kinematics of Tropical Rainfall Systems Ground Tracked at Mesoscale with Gages: First Results from the Campaigns 1999-2006 on the Upper Ouémé Valley (Benin). Journal of Hydrology, 375, 143-160. https://doi.org/10.1016/j.jhydrol.2009.01.011

[21] Moumouni, S., Gosset, M. and Houngninou, E. (2008) Main Features of Rain Drop 
Size Distributions Observed in Benin, West Africa, with Optical Disdrometers. Geophysical Research Letters, 35, L23807. http://dx.doi.org/10.1029/2008GL035755

[22] Zahiri, E-P., Bamba, I., Famien, A.M., Koffi, A.K. and Ochou, A.D. (2016) Mesoscale Extreme Rainfall Events in West Africa: The Cases of Niamey (Niger) and the Upper Ouémé Valley (Benin). Weather and Climate Extremes, 13, 15-34. https://doi.org/10.1016/j.wace.2016.05.001

[23] Peters, G., Fisher, B. and Clemens, M. (2010) Rain Attenuation of Radar Echoes Considering Finite-Range Resolution and Using Drop Size Distribution. Journal of Atmospheric and Oceanic Technology, 27, 829-842. https://doi.org/10.1175/2009JTECHA1342.1

[24] Maahn, M. and Kollias, P. (2012) Improved Micro Rain Radar Snow Measurements Using Doppler Spectra Post-Processing. Atmospheric Measurement Techniques, 5, 2661-2673. https://doi.org/10.5194/amt-5-2661-2012

[25] Merker, C., Peters, G., Clemens, M., Lengfeld, K. and Ament, F. (2015) A Novel Approach for Absolute Radar Calibration: Formulation and Theoretical Validation. Atmospheric Measurement Techniques, 8, 2521-2530. https://doi.org/10.5194/amt-8-2521-2015

[26] Lebel, T., Parker, D.J., Flamant, C., Bourlès, B., Marticorena, B., Mougins, E., Peugeot, C., Diedhiou, A., Haywood, J.M., Ngamini, J.B., Polcher, J., Redelsperger, J.L. and Thorncroft, C.D. (2010) The AMMA Field Campaigns: Multiscale and Multidisciplinary Observations in the West African Regions. Quarterly Journal of the Royal Meteorological Society, 136, 8-33. https://doi.org/10.1002/qj.486

[27] Strauch, R.G, Campbell, W.C., Chadwick, R.B. and Moran, K.B. (1976) Microwave FM-CW Doppler Radar for Boundary Layer Probing. Geophysical Research Letters, 3, 193-196. https://doi.org/10.1029/GL003i003p00193

[28] Atlas, D., Srivastava, R.C. and Sekhon, R.S. (1973) Doppler Radar Characteristics of Precipitation at Vertical Incidence. Reviews of Geophysics Reviews of Geophysics, 11, 1-35. https://doi.org/10.1029/RG011i001p00001

[29] Ulbrich, C.W. (1983) Natural Variations in the Analytical Form of the Raindrop Size Distribution. Journal of Applied Meteorology and Climatology, 22, 1764-1775. https://doi.org/10.1175/1520-0450(1983)022<1764:NVITAF $>2.0 . C O ; 2$

[30] Russell, B., Williams, E.R., Gosset, M., Cazenave, F., Descroix, L., Guy, N., Lebel, T., Ali, A., Metayer, F. and Quentin, G. (2010) Radar/Rain-Gauge Comparisons on Squall Lines in Niamey, Niger for the AMMA. Quarterly Journal of the Royal Meteorological Society, 136, 289-303. https://doi.org/10.1002/qj.548

[31] Delrieu, G., Creutin, J.D. and Saint-Andre, I. (1991) Relationships: Practical Results for Typical Weather Radar Wavelengths. Journal of Atmospheric and Oceanic Technology, 8, 467-476. https://doi.org/10.1175/1520-0426(1991)008<0467:MRRPRF>2.0.CO;2

[32] Hitschfeld, W. and Bordan, J. (1954) Errors Inherent in the Radar Measurement of Rainfall at Attenuating Wavelengths. Journal of the Atmospheric Sciences, 11, 58-67.

[33] Schneebeli, M. and Berne, A. (2012) An Extended Kalman Filter Framework for Polarimetric X-Band Weather: Radar Data Processing. Journal of Atmospheric and Oceanic Technology, 29, 711-730. https://doi.org/10.1175/JTECH-D-10-05053.1

[34] Zhang, G., Vivekanandan, J. and Brandes, E. (2001) A Method for Estimating Rain Rate and Drop Size Distribution from Polarimetric Radar Measurements. IEEE Transactions on Geoscience and Remote Sensing, 39, 830-841.

https://doi.org/10.1109/36.917906

[35] Andsager, K., Beard, K.V. and Laird, N.F. (1999) Laboratory Measurements of Axis Ratios for Large Raindrops. Journal of the Atmospheric Sciences, 56, 2673-2683. 
https://doi.org/10.1175/1520-0469(1999)056<2673:LMOARF>2.0.CO;2

[36] Peters, G., Fischer, B., Münster, H., Clemens, M. and Wagner, A. (2005) Profiles of Raindrop Size Distributions as Retrieved by Microrain Radars. Journal of Applied Meteorology and Climatology, 44, 1930-1949. https://doi.org/10.1175/JAM2316.1

[37] Rosenfeld, D. and Ulbrich, C.W. (2003) Cloud Microphysical Properties, Processes and Rainfall Estimation Opportunities. Meteorological Monographs, 30, 237-237. https://doi.org/10.1175/0065-9401(2003)030<0237:CMPPAR >2.0.CO;2

[38] Tokay, A., Kruger, A. and Krajewski, W.F. (2001) Comparison of Drop Size Distribution Measurements by Impact and Optical Disdrometers. Journal of Applied Meteorology and Climatology, 40, 2083-2097. https://doi.org/10.1175/1520-0450(2001)040<2083:CODSDM>2.0.CO;2

[39] Campos, E. and Zawadzki, I. (2000) Instrumental Uncertainties in $Z-R$ Relations. Journal of Applied Meteorology and Climatology, 39, 1088-1102. https://doi.org/10.1175/1520-0450(2000)039<1088:IUIZRR>2.0.CO;2

[40] Gould, N., Orban, D. and Toint, P. (2005) Numerical Methods Large-Scale Nonlinear Optimization. Acta Numerica, 14, 299. https://doi.org/10.1017/S0962492904000248

[41] Coleman, T.F. and Li, Y. (1994) On the Convergence of Interior-Reflective Newton Methods for Nonlinear Minimization Subject to Bounds. Mathematical Programming, 67, 189-224. https://doi.org/10.1007/BF01582221

[42] Coleman, T.F. and Li, Y. (1996) An Interior Trust Region Approach for Nonlinear Minimization Subject to Bounds. SIAM Journal on Optimization, 6, 418-445. https://doi.org/10.1137/0806023

[43] Hu, Z. and Srivastava, R.C. (1995) Evolution of Raindrop Size Distribution by Coalescence, Breakup and Evaporation: Theory and Observations. Journal of the Atmospheric Sciences, 52, 1761-1783.

[44] Marzuki, Hashiguchi, H., Shimomai, T., Rahayu, I., Vonnisa, M. and Afdal (2016) Performance Evaluation of Micro Rain Radar over Sumatra through Comparison with Didrometer and Wind Profiler. Progress in Electromagnetics Research M, 50, 33-46. https://doi.org/10.2528/PIERM16072808

[45] Tridon, F., Van Baelen, J. and Pointin, Y. (2011) Aliasing in Micro Rain Radar Data Due to Strong Vertical Winds. Geophysical Research Letters, 38, 4-7.

https://doi.org/10.1029/2010GL046018

[46] Low, T.B. and List, R. (1982) Collision, Coalescence and Breakup of Raindrops. Part I: Experimentally Established Coalescence Efficiencies and Fragment Size Distributions in Breakup. Journal of the Atmospheric Sciences, 39, 1591-1606. https://doi.org/10.1175/1520-0469(1982)039<1591:CCABOR>2.0.CO;2

[47] Srivastava, R.C. (1971) Size Distribution of Raindrops Generated by Their Breakup and Coalescence. Journal of the Atmospheric Sciences, 28, 410-415. https://doi.org/10.1175/1520-0469(1971)028<0410:SDORGB >2.0.CO;2

[48] Lee, G. (2006) Sources of Errors in Rainfall Measurements by Polarimetric Radar: Variability of Drop Size Distributions, Observational Noise and Variation of Relationships between $\mathrm{R}$ and Polarimetric Parameters. Journal of Atmospheric and Oceanic Technology, 23, 1005-1028. https://doi.org/10.1175/JTECH1899.1

[49] Kacou, M. (2014) Analyse des précipitations en zone sahélienne à partir d'un radar bande X polarimétrique. $\mathrm{PhD}$ Thesis, Université de Toulouse, Toulouse.

[50] Zahiri, E.P., Gosset, M., Lafore, J.P. and Gouget, V. (2008) Use of Radar Simulator on the Output Field from a Numerical Mesoscale Model to Analyze X-Band Rain Estimators. Journal of Atmospheric and Oceanic Technology, 25, 341-367. 
https://doi.org/10.1175/2007JTECHA933.1

[51] Zhao, K., Huang, H., Wang, M., Lee, W.-C., Chen, G., Wen, L., Wen, J., Zhang, G., Xue, M., Yang, Z., Liu, L., Wu, C., Hu, Z. and Chen, S. (2019) Recent Progress in Dual-Polarization Radar Research and Applications in China. Advances in Atmospheric Sciences, 36, 961-974,

[52] Anagnostou, M.N., Kalogiros, J., Marzano, F.S., Anagnostou, E.N., Montopoli, M. and Picciotti, E. (2013) Performance Evaluation of a New Dual-Polarization Microphysical Algorithm Based of Long-Term X-Band Radar and Disdrometer Observations. Journal of Hydrometeorology, 14, 560-576.

https://doi.org/10.1175/JHM-D-12-057.1

[53] Kalogiros, J., Anagnostou, M.N., Anagnostou, E.N., Montopoli, M., Picciotti, E. and Marzano, F.S. (2012) Optimum Estimation of Rain Microphysical Parameters Using $\mathrm{X}$-Band Dual-Polarization Radar Observables. IEEE Transactions on Geoscience and Remote Sensing, 51, 3063-3076. https://doi.org/10.1109/TGRS.2012.2211606 